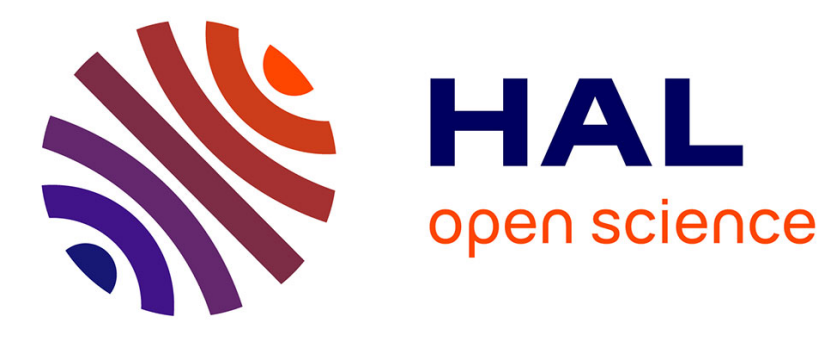

\title{
Ruin problems with worsening risks or with infinite mean claims
}

Dominik Kortschak, Stéphane Loisel, Pierre Ribereau

\section{To cite this version:}

Dominik Kortschak, Stéphane Loisel, Pierre Ribereau. Ruin problems with worsening risks or with infinite mean claims. Stochastic Models, 2014, 31 (1), pp.119-152. hal-00735843

\section{HAL Id: hal-00735843 \\ https://hal.science/hal-00735843}

Submitted on 27 Sep 2012

HAL is a multi-disciplinary open access archive for the deposit and dissemination of scientific research documents, whether they are published or not. The documents may come from teaching and research institutions in France or abroad, or from public or private research centers.
L'archive ouverte pluridisciplinaire HAL, est destinée au dépôt et à la diffusion de documents scientifiques de niveau recherche, publiés ou non, émanant des établissements d'enseignement et de recherche français ou étrangers, des laboratoires publics ou privés. 


\title{
Ruin problems with worsening risks or with infinite mean claims
}

\author{
Dominik Kortschak*, Stéphane Loisel*, Pierre Ribereau* \\ *Université de Lyon, Université Claude Bernard Lyon 1, Institut de Science \\ Financière et d'Assurances, 50 Avenue Tony Garnier, F-69007 Lyon, France
}

\begin{abstract}
In this paper, we obtain asymptotic ruin probabilities in two models where claim amounts become more and more adverse, because of phenomena like climate change or some kind of sectorial inflation. The method we use also enables us to study a risk model in which claims have infinite mean. In such models, ruin probability can be controlled by a strong increase in the premium income rate, which causes premium to become unacceptable for customers. We provide numerical illustrations of the impact of the (uncertain) speed of change in the parameter of the claim size distribution, both in terms of ruin and in terms of time at which premium becomes too high.
\end{abstract}

Keywords: Pareto distribution, regularly varying distributions, infinite mean, non homogenous risk process, ruin probability, asymptotics, climate change.

\section{Introduction}

Insurers are concerned that climate change is likely to increase the frequency and severity of some natural catastrophes, in particular hurricanes and floods. For other large non-life risks, sectorial inflation might also modify the claim severity tail in an adverse way. As loss distributions for those risks are already heavy-tailed, and as the exposure increases too at the same time (risk exposure doubles every 20 years in some regions of Florida for example), one may fear that a large claim causes ruin of the insurer, or that those risks become uninsurable because the premium required to maintain the solvency probability high enough becomes too large and unacceptable for policyholders. The same reasoning would apply at the reinsurance level. The type of effect of global warming on the claim frequency and severity remains uncertain. In the classical risk model, one way to increase the severity and frequency of natural catastrophes is to assume that the claim size distribution changes over time in a unfavorable direction.

For the sake of simplification, even if we prove some results in a more general setting, let us focus in this introduction on the compound Poisson model with Pareto distributed claim amounts. There are two main ways to obtain worsening risks: either one changes the shape parameter $\alpha$, which means that the distribution of claim amounts becomes more and more heavy-tailed, or one changes the scale parameter, which means that the claim distribution remains regularly varying with the same $\alpha$, but the average claim amount is multiplied by a coefficient that increases with time. We do not consider in the present 
paper any change in the claim frequency. In some cases, a simple time change might enable one to come back to the classical case. We will assume that premiums are adjusted immediately. In practice however the premiums will only be updated after some lag. If this lag is short enough our model will be a good approximation, but if the lag is too large one should consider models with a different premium income process.

In this paper, we study the asymptotics of the ruin probability with large initial surplus $u$ in the first case, when $\alpha_{t}$ tends to 1 as $t$ goes to infinity. The techniques are similar to the ones that we use to study the case where claim amounts are i.i.d. with infinite mean $(\alpha<1)$. When $\alpha<1$, the ruin probability is always 1 if one uses a constant premium income rate. But one may make the probability of ruin as small as one wants by increasing the premium income rate over time fast enough. This corresponds to a case where the probability of ruin can be made as small as one wants, even if the net profit condition is violated at any instant (because the premium income rate is finite and the average claim amount is infinite).

Of course, this result is of theoretical nature. In practice, the company would close its business after a certain time, when the premium income rate required for solvency reason would hit the maximum acceptable premium income rate level. In the present paper, we study the time at which risks could become uninsurable according to several global warming scenarios or different sectorial inflation scenarios, corresponding to different speeds of increase of claim severities. We carry out a similar study in the second case, where the scale parameter changes over time (instead of the shape parameter). We compare the asymptotics of ruin probability for large $u$ in the two cases. For risks that are already very dangerous (for $1<\alpha<2$, with infinite variance and finite mean), change in the scale parameter is the most dangerous. For risks that are less heavy-tailed at the beginning (with finite variance), change in the shape parameter is the most dangerous. This is consistent with the fact that premiums computed thanks to a ruin probability approach are often similar to premiums calculated with the variance principle (see e.g. [11]). For $\alpha_{0}>2$, claim amounts variance becomes infinite after a certain time in the first case, while it remains finite at any instant in the second case (even if it tends to infinity). Consequently, it is intuitive that the premium required to maintain a certain ruin probability constraint should increase faster in the first case than in the second one. We are able to show that it is the case for $u$ large enough.

Those results are useful from a researchwise dynamic risk management point of view. From a more practical point of view, insurers who are concerned with insurability of large risks would more care about the difference between the 1-year Value-at-Risk of the loss and the expected loss, which drives Solvency Capital Requirements in Solvency II (the new European regulation framework). Note that this SCR would be easy to compute in the two first models, but not in the infinite case because the average aggregate claim is infinite! In a numerical application section, we analyze the impact of different global warming effects in different scenarios, particularly in terms of waiting time before either ruin occurs or risks become uninsurable because the required safety loading would become too high. 
From a mathematical point of view, we are considering time inhomogeneous risk models. There is a huge amount of literature on non-homogeneous risk models. These models are mainly motivated by periodic effects (see e.g. [4, 3, $5,5,2]$ ). In the heavy-tailed case, these papers usually use some recursive method. The second type of motivation comes from inflation or investment (e.g. [17, 14, 15) ) where in the heavy tailed case it is often used that the risk is decreasing over time. [17] also allows for an increase in risk over time (motivated by wealth growth), but assumes that investments outweigh these effects. We should note that these two frameworks differ from our situation since we assume that risk increases over time. In terms of worsening risks, [8] considers a discrete risk model and obtains recursive methods for finite time ruin probabilities. [6] compute the moments of aggregate claims in a model where jump instant and sizes present some particular kind of dependence and where limited positive sectorial inflation can prevail over discounting effects. Infinite mean risks have been investigated in several papers related to operational risk, see e.g. [16].

Our paper is structured as follows: in Section 2, we state two models and compare the corresponding asymptotic risk ruin probabilities. We recall some definitions about regularly varying distributions in Section 3 . Then we introduce change in the shape parameter for the first model in Section 4 for the first model and a linear drift for the second model in Section 5. Finally, we present in Section 6 some simulations results on the two models.

\section{Two specific models}

As mentioned above we want to study a risk process, when the underlying risk changes over time. Concretely let $N_{t}$ be a Poisson process with intensity $\lambda$ and $X_{t}$ are independent Pareto distributed random variables with distribution $\bar{F}_{t}=\left(1+x / d_{t}\right)^{-\alpha_{t}}$ where the change of the distribution over time is characterized by

$$
\mathbb{E}\left[X_{t}\right]=\frac{d}{\alpha_{0}-1}\left(1+c_{\alpha} t\right)
$$

We consider the risk process $(\rho>0)$

$$
R_{t}=u+\int_{0}^{t}(1+\rho) \lambda \mathbb{E}\left[X_{t}\right] \mathrm{d} t-\sum_{i=1}^{N_{t}} X_{\mathcal{T}_{i}}=u+\frac{(1+\rho) \lambda d\left(1+c_{\alpha} t\right)^{2}}{2 c_{\alpha}\left(\alpha_{0}-1\right)}-\sum_{i=1}^{N_{t}} X_{\mathcal{T}_{i}},
$$

where $\mathcal{T}_{i}$ is the time of the $i$-th jump. Further we denote with $S_{t}=\sum_{i=1}^{N_{t}} X_{\mathcal{T}_{i}}$ and

$$
p(t)=\frac{(1+\rho) \lambda d\left(1+c_{\alpha} t\right)^{2}}{2 c_{\alpha}\left(\alpha_{0}-1\right)} .
$$

We now study two sets of parameters that assure that (1) holds. In the first model we change the parameter $\alpha$ which means that the distribution of $X_{t}$ gets more and more heavy tailed. In this case we have that $\bar{F}_{t}^{(1)}(x)=(1+x / d)^{-\alpha_{t}}$ where

$$
\alpha_{t}=\frac{\alpha_{0}-1}{1+c_{\alpha} t}+1
$$


we will call this variant model 1 . An obvious alternative to this is model 2 where we only change the other parameter. i.e. we choose $\bar{F}_{t}^{(2)}(x)=\left(1+x / d_{t}\right)^{-\alpha_{0}}$ where

$$
d_{t}=d\left(1+c_{\alpha} t\right)
$$

We now want to compare these two models. An obvious method therefore is to consider the ruin probability $\psi^{(i)}(u)=\mathbb{P}\left(\inf _{t>0} R_{t}^{(i)} \leq 0\right)$. Denote with $\mu=\mathbb{E}\left[X_{0}\right]=\frac{d}{\alpha_{0}-1}$. We get by Theorems 4.2 respectively Corollary 5.2 that

$$
\begin{aligned}
\psi^{(1)}(u) & \sim \lambda \sqrt{\frac{2 u\left(\alpha_{0}-1\right)}{(1+\rho) \lambda c_{\alpha}}}(1+u / d)^{-1} \int_{0}^{\infty}\left(1+t^{2}\right)^{-1} \mathrm{~d} t \sim \frac{\pi d u^{-0.5}}{2} \sqrt{\frac{2\left(\alpha_{0}-1\right)}{(1+\rho) \lambda c_{\alpha}}} . \\
\psi^{(2)}(u) & \sim \frac{\lambda}{c_{\alpha}} \sqrt{u}\left(1+\frac{\sqrt{u}}{d}\right)^{-\alpha_{0}} \int_{0}^{\infty}\left(\frac{1}{t}+\frac{\rho \lambda \mu}{2 c_{\alpha}} t\right)^{-\alpha_{0}} \mathrm{~d} t \\
& \sim \frac{\lambda}{c_{\alpha}} d^{\alpha_{0}} u^{-\frac{\alpha_{0}-1}{2}} \int_{0}^{\infty}\left(\frac{1}{t}+\frac{\rho \lambda \mu}{2 c_{\alpha}} t\right)^{-\alpha_{0}} \mathrm{~d} t .
\end{aligned}
$$

An interesting fact about model 1 is that we can even choose a premium rate that is less than the mean of the claims and the ruin probability is still finite. Further note that for $\alpha_{0}<2$ the shape change asymptotic is less risky and is the opposite for $\alpha_{0}>2$. For $\alpha_{0}=2$ this can be decided by

$$
\int_{0}^{\infty}\left(\frac{1}{t}+\frac{\rho \lambda \mu}{2 c_{\alpha}} t\right)^{-\alpha_{0}} \mathrm{~d} t=\frac{\pi}{4}\left(\frac{\rho \lambda \mu}{2 c_{\alpha}}\right)^{-3 / 2}=\frac{\pi}{4}\left(\frac{\rho \lambda d}{2 c_{\alpha}\left(\alpha_{0}-1\right)}\right)^{-3 / 2} .
$$

\section{Regularly varying distributions}

The main motivation of this paper is to study the effect of risk theory in a time changing environment motivated by climate change. Since there are different ways how risk can change over time we used two different models, where the claims are Pareto distribution. One of the main properties of the Pareto distribution is that the tail behaves asymptotically like a power. The class of functions that behave asymptotically like a power is the class of regularly varying functions. A function $f$ is regularly varying with index $-\alpha$ if

$$
\lim _{x \rightarrow \infty} \frac{f(a x)}{f(x)}=a^{-\alpha}
$$

for a distributions with tail $\bar{F}(x)$ that is regularly varying with index $-\alpha$ we also say that the distribution is regularly varying with index $\alpha$.

In the following sections we will replace the Pareto distribution by certain regularly varying distributions to gain more generality.

We will list some properties of regularly varying distributions that will be useful later in the paper and basically show how we can replace a regularly varying function with a power function. All results can for example be found in [7].

One of the most famous properties of regularly varying distributions is Karamata's Theorem. It states that for $\alpha>1$

$$
\int_{x}^{\infty} f(x) \mathrm{d} x \sim \frac{x}{\alpha-1} f(x)
$$


Since a regularly varying distribution behaves asymptotically like a power, one would guess that it is close to a monotone function. Indeed this guess is true and for $\alpha>0$ we get that

$$
\lim _{x \rightarrow \infty} \frac{\sup _{t>x} f(t)}{f(x)}=1 .
$$

When interchanging limits and integration, one often needs bounds for values $f(x) / f(y)$. Such bounds are called Potter bounds: for $f$ regularly varying with index $-\alpha$, and for any chosen $\delta>0$ and $\epsilon>0$, there exists $x_{0}$ such that for all $x, y>x_{0}$

$$
\frac{f(x)}{f(y)} \leq(1+\delta) \max \left(\left(\frac{x}{y}\right)^{-\alpha+\epsilon},\left(\frac{x}{y}\right)^{-\alpha-\epsilon}\right) .
$$

\section{Change in the shape parameter}

In this section we consider the first model. In the first model the parameter $\alpha \rightarrow 1$ as $t \rightarrow \infty$. This means that the distribution of the claims as $t \rightarrow \infty$ converges to a Pareto distribution with parameter $\alpha=1$. Since for large initial capital $u$ one assumes that if ruin occurs it occurs rather late, we guess that the ruin probability in the first model will behave asymptotically like in a model with i.i.d. claims that are distributed with the limit distribution. The proof of this guess can be split in two parts. In Subsection 4.1 we provide the asymptotic of a ruin probability with i.i.d. claims that have infinite mean. In Subsection 4.2 we then show that under some assumptions the infinite mean model can be used as an upper bound. Together with a corresponding lower bound, the guess can be shown to be true by standard calculations.

\subsection{Ruin probability for a risk process with infinite mean}

In this section we considering the following risk process.

$$
R_{t}=R_{t}(u)=u+p(t)-\sum_{i=1}^{N_{t}} X_{i}
$$

where the $X_{i}$ are i.i.d. with distribution function $F, N_{t}$ is a Poisson process with intensity $\lambda$ and $p(t)$ are the premiums collected up to time $t$. We are interested in the infinite time ruin probability

$$
\psi(u)=\mathbb{P}\left(\inf _{t \geq 0} R_{t}=0\right) .
$$

Theorem 4.1. If $X_{1}, X_{2}, \ldots$ are i.i.d. random variables with distribution $F(x)$ that is regularly varying with index $0<\alpha \leq 1$, and regularly varying density $f(x)$. If further $p(T)$ is regularly varying with index $\beta>1 / \alpha$ (continuous and strict monotonic increasing) then

$$
\psi(u) \sim \lambda \int_{0}^{\infty} \bar{F}(u+p(T)) \mathrm{d} T \sim \lambda p^{-1}(u) \bar{F}(u) \int_{0}^{\infty}\left(1+t^{\beta}\right)^{-\alpha} \mathrm{d} t
$$

Proof. Throughout the proof we will denote with $c_{i}$ a constant whose actual value is not important. At first note that by substitution $t=T p^{-1}(u)$

$$
\int_{0}^{\infty} \bar{F}(u+p(T)) \mathrm{d} T=p^{-1}(u) \bar{F}(u) \int_{0}^{\infty} \frac{\bar{F}\left(u\left(1+\frac{p\left(t p^{-1}(u)\right)}{p\left(p^{-1}(u)\right)}\right)\right)}{\bar{F}(u)} \mathrm{d} t
$$


Since for some $0<\epsilon<(\alpha+\beta) / 2-\sqrt{(\alpha-\beta)^{2} / 4+1}$ we get by Potter bounds [7]

$$
\frac{\bar{F}\left(u\left(1+\frac{p\left(t p^{-1}(u)\right)}{p\left(p^{-1}(u)\right)}\right)\right)}{\bar{F}(u)} \leq c_{1}\left(1+\frac{p\left(t p^{-1}(u)\right)}{p\left(p^{-1}(u)\right)}\right)^{-\alpha+\epsilon} \leq c_{1}\left(1+c_{2} t^{\beta-\epsilon}\right)^{-\alpha+\epsilon}
$$

and the last function is integrable we get by dominated convergence that

$$
\lambda \int_{0}^{\infty} \bar{F}(u+p(T)) \mathrm{d} T \sim \lambda p^{-1}(u) \bar{F}(u) \int_{0}^{\infty}\left(1+t^{\beta}\right)^{-\alpha} \mathrm{d} t .
$$

By the Marcinkiewicz-Zygmund strong law of large numbers (c.f. [10, Theorem 2.1.5]) and $N_{t} \sim \lambda t$ a.s. we get that

$$
p(t)-\sum_{i=1}^{N_{t}} X_{i} \sim p(t) \quad \text { a.s.. }
$$

From Lemma B.1 and the fact that $F$ is regularly varying we get that the asymptotic is an asymptotic lower bound. So we only have to show that it is an upper bound too. We will denote with $F^{* n}$ respectively $f^{* n}$ the distribution or respectively the density of the $n$-times convolution of $F$.

By conditioning on the number of jumps that lead to ruin we get that

$$
\begin{aligned}
\psi(u)= & \int_{0}^{\infty} \lambda e^{-\lambda s} \bar{F}(p(s)+u) \mathrm{d} s \\
& +\sum_{n=2}^{\infty} \mathbb{E}\left[\bar{F}\left(u+p\left(\mathcal{T}_{n}\right)-\sum_{i=1}^{n-1} X_{i}\right) 1_{\left\{\sum_{i=1}^{k} X_{i} \leq p\left(\mathcal{T}_{k}\right)+u: k \leq(n-1)\right\}}\right] .
\end{aligned}
$$

For $S_{n-1}=\sum_{i=1}^{n-1} X_{i}$ and $\epsilon>0$ we get that

$$
\begin{aligned}
& \mathbb{E}\left[\bar{F}\left(u+p\left(\mathcal{T}_{n}\right)-\sum_{i=1}^{n-1} X_{i}\right) 1_{\left\{\sum_{i=1}^{k} X_{i} \leq p\left(\mathcal{T}_{k}\right)+u: k \leq(n-1)\right\}}\right] \\
& \leq \mathbb{E}\left[\bar{F}\left(u+p\left(\mathcal{T}_{n}\right)-S_{n-1}\right) 1_{\left\{S_{n-1} \leq p\left(\mathcal{T}_{n-1}\right)+u\right\}}\right] \\
& \leq \mathbb{E}\left[\bar{F}\left(u+p\left(\mathcal{T}_{n}\right)-S_{n-1}\right) 1_{\left\{S_{n-1} \leq \epsilon\left(u+p\left(\mathcal{T}_{n}\right)\right)\right\}}\right] \\
& \quad+\mathbb{E}\left[\bar{F}\left(u+p\left(\mathcal{T}_{n}\right)-S_{n-1}\right) 1_{\left\{\epsilon\left(u+p\left(\mathcal{T}_{n}\right)\right)<S_{n-1} \leq u+p\left(\mathcal{T}_{n}\right)\right\}}\right] \\
& =I_{1}+I_{2} .
\end{aligned}
$$

Since $\mathcal{T}_{n}$ follows an Erlang distribution we get that

$$
I_{1} \leq \mathbb{E}\left[\bar{F}\left((1-\epsilon)\left(u+p\left(\mathcal{T}_{n}\right)\right)\right)\right]=\int_{0}^{\infty} \frac{\lambda^{n} T^{n-1}}{(n-1) !} e^{-\lambda T} \bar{F}((1-\epsilon)(u+p(T))) \mathrm{d} T .
$$

Further we have that

$$
\sum_{n=1}^{\infty} \int_{0}^{\infty} \frac{\lambda^{n} T^{n-1}}{(n-1) !} e^{-\lambda T} \bar{F}((1-\epsilon)(u+p(T))) \mathrm{d} T=\int_{0}^{\infty} \lambda \bar{F}((1-\epsilon)(u+p(T))) \mathrm{d} T
$$




$$
\sim(1-\epsilon)^{-\alpha} \int_{0}^{\infty} \lambda \bar{F}(u+p(T)) \mathrm{d} T
$$

It remains to show that $\sum_{n=2}^{\infty} I_{2}=o(\psi(u))$. Note that

$$
\begin{aligned}
I_{2} \leq & \mathbb{E}\left[\bar{F}\left(u+p\left(\mathcal{T}_{n}\right)-S_{n-1}\right) 1_{\left\{\epsilon\left(u+p\left(\mathcal{T}_{n}\right)\right)<S_{n-1} \leq u+p\left(\mathcal{T}_{n}\right)\right\}} 1_{\left\{\mathcal{T}_{n} \leq \frac{n}{2 \lambda}\right\}}\right] \\
& +\mathbb{E}\left[\bar{F}\left(u+p\left(\mathcal{T}_{n}\right)-S_{n-1}\right) 1_{\left\{\epsilon\left(u+p\left(\mathcal{T}_{n}\right)\right)<S_{n-1} \leq u+p\left(\mathcal{T}_{n}\right)\right\}} 1_{\left\{\mathcal{T}_{n}>\frac{n}{2 \lambda}\right\}}\right] \\
= & J_{1}(n)+J_{2}(n) .
\end{aligned}
$$

By Lemma B.2 we get that there exists $c_{2}>0$ with

$$
J_{1}(n) \leq \mathbb{P}\left(\mathcal{T}_{n} \lambda \leq \frac{n}{2 \lambda}\right) \leq e^{-c_{2} n} .
$$

Hence there exists a constant $c_{3}$ such that

$$
\sum_{n=-c_{3} \log (\bar{F}(u))}^{\infty} J_{1}(n)=o(\bar{F}(u))=o(\psi(u)) .
$$

Since $J_{1}(n) \leq \mathbb{P}\left(S_{n}>u\right) \leq \bar{F}(u / n)$ we have that

$$
\sum_{n=1}^{-c_{3} \log (\bar{F}(u))} J_{1}(n) \leq \sum_{n=1}^{-c_{3} \log (\bar{F}(u))} \bar{F}(u / n) \leq c_{3} \log (\bar{F}(u)) \bar{F}\left(\frac{u}{c_{3} \log (\bar{F}(u))}\right)=o(\psi(u)) .
$$

We finally have to consider $J_{2}$. From Corollary A.1 we get that there exist $c_{4}>0$ and $u>u_{\epsilon}$ (for some $\left.u_{\epsilon}>0\right)$, all $n>1, \mathcal{T}_{n}>n /(2 \lambda)$ and $x>\epsilon\left(u+p\left(\mathcal{T}_{n}\right)\right)$

$$
\mathbb{P}\left(S_{n-1} \in(x, x+1]\right) \leq c_{4} n \mathbb{P}\left(X_{n} \in(x, x+1]\right) .
$$

With Lemma B.3 we get that for some $c_{5}>0$ (that does not depend on $n$ )

$$
\begin{aligned}
& \mathbb{P}\left(S_{n}>u+p\left(\mathcal{T}_{n}\right), \epsilon\left(u+p\left(\mathcal{T}_{n}\right)\right)<S_{n-1} \leq u+p\left(\mathcal{T}_{n}\right), \mathcal{T}_{n}>\frac{n}{2 \lambda}\right) \\
& \leq \mathbb{E}\left[c_{5} n f\left(u+p\left(\mathcal{T}_{n}\right)\right) \int_{0}^{u+p\left(\mathcal{T}_{n}\right)} \bar{F}(x) \mathrm{d} x, 1_{\left\{\mathcal{T}_{n}>\frac{n}{2 \lambda}\right\}}\right] \\
& =o\left(\mathbb{E}\left[\bar{F}\left(u+p\left(\mathcal{T}_{n}\right)\right)\right]\right)
\end{aligned}
$$

where the last equality follows by assumption $\left(f(p(t)) \int_{0}^{p(t)} \bar{F}(x) \mathrm{d} x\right.$ is regularly varying with index $-2 \alpha \beta<-\alpha \beta-1$ ) and is meant as $u \rightarrow \infty$ (uniformly in $n$ and $T>n / 2 \lambda$ ). Hence we get that

$$
\begin{aligned}
\sum_{n=2}^{\infty} J_{2}(n) & =o(1) \sum_{n=1}^{\infty} \int_{n /(2 \lambda)}^{\infty} \frac{\lambda^{n} T^{n-1}}{(n-1) !} e^{-\lambda T} \bar{F}(u+p(T)) \mathrm{d} T \\
& \leq o(1) \sum_{n=1}^{\infty} \int_{0}^{\infty} \frac{\lambda^{n} T^{n-1}}{(n-1) !} e^{-\lambda T} \bar{F}(u+p(T)) \mathrm{d} T
\end{aligned}
$$




$$
=o(1) \lambda \int_{0}^{\infty} \bar{F}(u+p(T)) \mathrm{d} T \text {. }
$$

Since

$$
\int_{0}^{\infty} \lambda e^{-\lambda s} \bar{F}(p(s)+u) \mathrm{d} s \leq \bar{F}(u)
$$

we get with $\epsilon \rightarrow 0$ that

$$
\psi(u) \lesssim \lambda \int_{0}^{\infty} \bar{F}(u+p(T)) \mathrm{d} T .
$$

\subsection{Bounds for the first model}

We now give two bounds for the ruin probability in certain risk models in a time changing environment. For the first model one can easily see that the assumptions of Theorem 4.2 are fulfilled and that the upper and lower bounds are asymptotically the same.

Theorem 4.2. Let $F$ be a distribution that is regularly varying with index $\alpha$ and regularly varying density $f(x)$. Assume that the premiums collected up to time $t(p(t))$ are regularly varying with index $\beta>1 / \alpha$.

Assume that $X_{t}$ is a family of independent random variables with distribution $F_{t}$ such that for all $x>0$ and $t>0 F_{t}(x) \geq F(x)$. Further assume that there exists $\beta>0$ such that for every $\delta>0$ there exists $x_{0}$ such that for $y>x>x_{0}$ and all $t>0$, we have $\bar{F}_{t}(y) \geq(1-\delta)(x / y)^{\beta} \bar{F}_{t}(x)$. Then for the risk process

$$
R_{t}=u+p(t)-\sum_{i=1}^{N_{t}} X_{\mathcal{T}_{i}}
$$

we have that

$$
\lambda \int_{0}^{\infty} \bar{F}_{t}(u+p(t)) \mathrm{d} t \lesssim \psi(u) \lesssim \lambda \int_{0}^{\infty} \bar{F}(u+p(t)) \mathrm{d} t .
$$

Proof. We will denote with $Y_{t} t \geq 0$ i.i.d. random variables with distribution $F$. By a coupling argument we can choose $Y_{t}>X_{t}$. Hence Theorem 4.1 proves the upper bound. For the lower bound note that by the Marcinkiewicz-Zygmund strong law of large numbers (c.f. [10, Theorem 2.1.5]) and $N_{t} \sim \lambda t$ a.s. we get that

$$
p(t)-\sum_{i=1}^{N_{t}} Y_{\mathcal{T}_{i}} \sim p(t) \quad \text { a.s. }
$$

and hence

$$
p(t)-\sum_{i=1}^{N_{t}} X_{\mathcal{T}_{i}} \sim p(t) \quad \text { a.s.. }
$$

From Lemma B.1 we get that for every $\epsilon>0$ and $\delta>0$ we get that for $u$ large enough

$$
\psi(u) \gtrsim(1-\epsilon) \lambda \int_{0}^{\infty} \bar{F}_{t}(u+K+(1+\epsilon) p(t)) \mathrm{d} t
$$




$$
\begin{aligned}
& \gtrsim(1-\epsilon)(1-\delta) \lambda \int_{0}^{\infty}\left(\frac{u+K+(1+\epsilon) p(t)}{u+p(t)}\right)^{-\beta} \bar{F}_{t}(u+p(t)) \mathrm{d} t \\
& =(1-\epsilon)(1-\delta) \lambda \int_{0}^{\infty}\left(1+\frac{K+\epsilon p(t)}{u+p(t)}\right)^{-\beta} \bar{F}_{t}(u+p(t)) \mathrm{d} t \\
& \gtrsim \frac{(1-\epsilon)(1-\delta)}{(1+\epsilon+K / u)^{\beta}} \lambda \int_{0}^{\infty} \bar{F}_{t}(u+p(t)) \mathrm{d} t .
\end{aligned}
$$

The Theorem follows with $\epsilon \rightarrow 0$ and $\delta \rightarrow 0$.

\section{$5 \quad$ Linear drift}

We now consider the second model. We will prove the result for regularly varying distributions. Therefore let $X_{i}$ be i.i.d. random variables with mean $\mu$. We will consider the risk process

$$
R_{t}=u+p(t)-\sum_{i=1}^{N_{t}} \mathcal{T}_{i} X_{i}
$$

where for some $\rho>0$

$$
p(t) \sim \frac{\lambda \mu+\rho}{2} t^{2} .
$$

Theorem 5.1. If $X_{1}, X_{2}, \ldots$ are i.i.d. random variables with distribution $F(x)$ that is regularly varying with index $\alpha>1$, mean $\mu$, and regularly varying density $f(x)$ then

$$
\psi(u) \sim \lambda \int_{0}^{\infty} \bar{F}\left(\frac{u}{t}+\frac{\rho}{2} t\right) \mathrm{d} t \sim \lambda \sqrt{u} \bar{F}(\sqrt{u}) \int_{0}^{\infty}\left(\frac{1}{t}+\frac{\rho}{2} t\right)^{-\alpha} \mathrm{d} t
$$

Proof. Since it is well known that $\lambda \mathcal{T}_{n} / n \rightarrow 1$ and $N_{t} / t \rightarrow \lambda$ a.s. it follows from Lemma B.5 that

$$
\sum_{i=1}^{n} \mathcal{T}_{i} X_{i} \sim \mu \frac{n^{2}}{2 \lambda} \quad \text { a.s. } \quad \text { and hence } \quad \sum_{i=1}^{N_{t}} \mathcal{T}_{i} X_{i} \sim \lambda \mu \frac{t^{2}}{2} \quad \text { a.s. }
$$

Together with Lemma B.1 and the fact that $\bar{F}(x)$ is regularly varying we get that (2) is a lower bound.

To prove that (2) is also an upper asymptotic bound first choose a $k>0$ and an $\epsilon<1 / k^{2}$ that fulfill the conditions of Lemma 5.3. Note that for a given $k, \epsilon$ can be taken arbitrary small. Further denote with $m_{n}=\lfloor(1-k \epsilon) n\rfloor$. Choose an $\epsilon_{1}$ such that

$$
1-\epsilon<\left(1-\epsilon_{1}\right)\left(1-\frac{2 \epsilon_{1}^{2}}{(1-k \epsilon)^{2}}\right)<\left(1+\epsilon_{1}\right)\left(1+\epsilon_{1}+\frac{3 \epsilon_{1}^{2}}{(1-k \epsilon)^{2}}\right)<1+\epsilon
$$

and define the sets

$$
A_{n}=\left\{\sup _{m \geq \epsilon_{1} n}\left|\frac{\lambda \mathcal{T}_{m}}{m}-1\right| \leq \epsilon_{1}\right\}
$$

Note that by Lemma B.2 there exists a $\kappa_{\epsilon}>0$ with

$$
\mathbb{P}\left(A_{n}^{c}\right) \leq \sum_{k=\left\lceil\epsilon_{1} n\right\rceil}^{\infty} \mathbb{P}\left(\left|\frac{\lambda \mathcal{T}_{k}}{k}-1\right|>\epsilon_{1}\right) \leq 2 \sum_{k=\left\lceil\epsilon_{1} n\right\rceil}^{\infty} e^{-\epsilon_{1} \kappa_{\epsilon} k} \leq \frac{2 e^{-\epsilon_{1} \kappa_{\epsilon} n}}{1-e^{-\kappa_{\epsilon}}}
$$


The main point of the sets $A_{n}$ is that there exists $n_{0}$ such that for all $n>n_{0}$ and $m \geq m_{n}$ and on these sets

$$
\begin{aligned}
\sum_{i=1}^{m} \mathcal{T}_{i} & \leq \sum_{i=1}^{\left\lceil\epsilon_{1} n\right\rceil-1} \mathcal{T}_{i}+\sum_{i=\left\lceil\epsilon_{1} n\right\rceil}^{m} \mathcal{T}_{i} \leq\left(1+\epsilon_{1}\right) \frac{\left(\left\lceil\epsilon_{1} n\right\rceil-1\right)\left\lceil\epsilon_{1} n\right\rceil}{\lambda}+\left(1+\epsilon_{1}\right) \sum_{i=\left\lceil\epsilon_{1} n\right\rceil}^{m} \frac{i}{\lambda} \\
& \leq \frac{1+\epsilon_{1}}{\lambda}\left(\left(\epsilon_{1} n+1\right) \epsilon_{1} n+\frac{m(m+1)}{2}\right)=\frac{\left(1+\epsilon_{1}\right) m^{2}}{2 \lambda}\left(1+\frac{2\left(\epsilon_{1} n+1\right) \epsilon_{1} n}{m^{2}}+\frac{1}{m}\right) \\
& \leq \frac{\left(1+\epsilon_{1}\right) m^{2}}{2 \lambda}\left(1+\frac{2\left(\epsilon_{1} n+1\right) \epsilon_{1} n}{\lfloor(1-k \epsilon) n\rfloor^{2}}+\frac{1}{\lfloor(1-k \epsilon) n\rfloor}\right) \\
& \leq \frac{\left(1+\epsilon_{1}\right) m^{2}}{2 \lambda}\left(1+\epsilon_{1} \frac{2\left(\epsilon_{1} n+1\right) n}{((1-k \epsilon) n-1)^{2}}+\frac{1}{(1-k \epsilon) n-1}\right) \\
& \leq \frac{m^{2}}{2 \lambda}\left(1+\epsilon_{1}\right)\left(1+\epsilon_{1}+\frac{3 \epsilon_{1}^{2}}{(1-k \epsilon)^{2}}\right) \leq(1+\epsilon) \frac{m^{2}}{2 \lambda} .
\end{aligned}
$$

and

$$
\begin{aligned}
\sum_{i=1}^{m} \mathcal{T}_{i} & \geq \sum_{i=\left\lceil\epsilon_{1} n\right\rceil}^{m} \mathcal{T}_{i} \geq\left(1-\epsilon_{1}\right) \sum_{i=\left\lceil\epsilon_{1} n\right\rceil}^{m} \frac{i}{\lambda}=\left(1-\epsilon_{1}\right) \frac{m^{2}}{2 \lambda}\left(1+\frac{1}{m}-\frac{\left\lceil\epsilon_{1} n\right\rceil\left(\left\lceil\epsilon_{1} n\right\rceil+1\right)}{m^{2}}\right) \\
& \geq\left(1-\epsilon_{1}\right) \frac{m^{2}}{2 \lambda}\left(1-\frac{\left(\epsilon_{1} n+2\right)\left(\epsilon_{1} n+1\right)}{((1-k \epsilon) n-1)^{2}}\right) \geq \frac{m^{2}}{2 \lambda}\left(1-\epsilon_{1}\right)\left(1-\frac{2 \epsilon_{1}^{2}}{(1-k \epsilon)^{2}}\right) \\
& \geq(1-\epsilon) \frac{m^{2}}{2 \lambda} .
\end{aligned}
$$

Now to prove the upper bound note that

$$
\begin{aligned}
\psi(u)= & \int_{0}^{\infty} \lambda e^{-\lambda s} \bar{F}\left(\frac{u+p(s)}{s}\right) \mathrm{d} s \\
& +\sum_{n=2}^{\infty} \mathbb{E}\left[\bar{F}\left(\frac{u+p\left(\mathcal{T}_{n}\right)-\sum_{i=1}^{n-1} \mathcal{T}_{i} X_{i}}{\mathcal{T}_{n}}\right) 1_{\left\{\sum_{i=1}^{k} \mathcal{T}_{i} x_{i} \leq p\left(\mathcal{T}_{k}\right)+u: k \leq(n-1)\right\}}\right] .
\end{aligned}
$$

Denote with $g(u)=\left\lceil\log \bar{F}(u)^{2}\right\rceil$ we have that

$$
\sum_{n=g(u)}^{\infty} \mathbb{P}\left(A_{n}^{c}\right) \leq \frac{2}{1-e^{-\kappa_{\epsilon}}} \sum_{n=g(u)}^{\infty} e^{-\epsilon_{1} \kappa_{\epsilon} n} \leq \frac{2}{1-e^{-\kappa_{\epsilon}}} e^{-\epsilon_{1} \kappa_{\epsilon} \log (\bar{F}(u))^{2}} \sum_{n=0}^{\infty} e^{-\epsilon_{1} \kappa_{\epsilon} n}=o(\psi(u))
$$

and

$$
\begin{aligned}
& \sum_{n=2}^{g(u)} \mathbb{E}\left[\bar{F}\left(\frac{u+p\left(\mathcal{T}_{n}\right)-\sum_{i=1}^{n-1} \mathcal{T}_{i} X_{i}}{\mathcal{T}_{n}}\right) 1_{\left\{\sum_{i=1}^{k} \mathcal{T}_{i} x_{i} \leq p\left(\mathcal{T}_{k}\right)+u: k \leq(n-1)\right\}}\right] \\
& \leq \sum_{n=2}^{g(u)} \mathbb{P}\left(\sum_{i=1}^{n} \mathcal{T}_{i} X_{i}>u+p\left(\mathcal{T}_{n}\right)\right) \leq g(u) \sum_{n=2}^{g(u)} \mathbb{E}\left[\bar{F}\left(\frac{u+p\left(\mathcal{T}_{n}\right)}{g(u) \mathcal{T}_{n}}\right)\right] \\
& \lesssim g(u)^{2+\alpha} \bar{F}\left(\sqrt{\frac{2 u}{\lambda \mu+\rho}}\right)=o(\psi(u)) .
\end{aligned}
$$


So we can concentrate on the sets $A_{n}$ and $n>g(u)$. For $n>\epsilon \sqrt{u}$ we get from Lemma 5.3 we get that there exists a function $o(\epsilon) \rightarrow 0$ as $\epsilon \rightarrow 0$

$$
\begin{aligned}
& \mathbb{E}\left[\bar{F}\left(\frac{u+p\left(\mathcal{T}_{n}\right)-\sum_{i=1}^{n-1} \mathcal{T}_{i} X_{i}}{\mathcal{T}_{n}}\right) 1_{\left\{\sum_{i=1}^{k} \mathcal{T}_{i} X_{i} \leq p\left(\mathcal{T}_{k}\right)+u: k \leq(n-1)\right\}} 1_{\left\{A_{n}\right\}}\right] \\
& \mathbb{E}\left[\bar{F}\left(\frac{u+p\left(\mathcal{T}_{n}\right)-\sum_{i=1}^{n-1} \mathcal{T}_{i} X_{i}}{\mathcal{T}_{n}}\right) 1_{\left\{\sum_{i=1}^{m_{n}} \mathcal{T}_{i} X_{i} \leq p\left(\mathcal{T}_{m_{n}}\right)+u, \sum_{i=1}^{n-1} \mathcal{T}_{i} X_{i} \leq p\left(\mathcal{T}_{n}\right)+u\right\}} 1_{\left\{A_{n}\right\}}\right] \\
& =\mathbb{E}\left[\mathbb{P}\left(\sum_{i=1}^{n} \frac{\mathcal{T}_{i}}{\mathcal{T}_{n}} X_{i}>\frac{u+p\left(\mathcal{T}_{n}\right)}{\mathcal{T}_{n}}, \sum_{i=1}^{m_{1}} \mathcal{T}_{i} X_{i} \leq u+p\left(\mathcal{T}_{m_{1}}\right), \sum_{i=1}^{n-1} \frac{\mathcal{T}_{i}}{\mathcal{T}_{n}} X_{i} \leq \frac{u+p\left(\mathcal{T}_{n}\right)}{\mathcal{T}_{n}} \mid \mathcal{T}\right) 1_{\left\{A_{n}\right\}}\right] \\
& \leq(1+o(\epsilon)) \mathbb{E}\left[\bar{F}\left(\frac{u+\frac{\rho}{2} \mathcal{T}_{n}^{2}}{\mathcal{T}_{n}}\right) 1_{\left\{A_{n}\right\}}\right] \\
& \leq(1+o(\epsilon)) \mathbb{E}\left[\bar{F}\left(\frac{u+\frac{\rho}{2} \mathcal{T}_{n}^{2}}{\mathcal{T}_{n}}\right)\right] .
\end{aligned}
$$

It follows that

$$
\begin{aligned}
\sum_{n=\epsilon \sqrt{u}}^{\infty} \mathbb{E}\left[\bar{F}\left(\frac{u+\frac{\rho}{2} \mathcal{T}_{n}^{2}}{\mathcal{T}_{n}}\right)\right] & \leq \sum_{n=1}^{\infty} \int_{0}^{\infty} \frac{\lambda^{n} t^{n-1}}{(n-1) !} e^{-\lambda t} \bar{F}\left(\frac{u+\frac{\rho}{2} t^{2}}{t}\right) \mathrm{d} t \\
& =\lambda \int_{0}^{\infty} \bar{F}\left(\frac{u+\rho \frac{t^{2}}{2}}{t}\right) \mathrm{d} t
\end{aligned}
$$

The last equality follows by standard arguments of regularly distributions.

Finally for $g(u)<n<\epsilon \sqrt{u}$ we get from Lemma 5.3

$$
\begin{aligned}
& \mathbb{E}\left[\bar{F}\left(\frac{u+p\left(\mathcal{T}_{n}\right)-\sum_{i=1}^{n-1} \mathcal{T}_{i} X_{i}}{\mathcal{T}_{n}}\right) 1_{\left\{\sum_{i=1}^{k} \mathcal{T}_{i} x_{i} \leq p\left(\mathcal{T}_{k}\right)+u: k \leq(n-1)\right\}} 1_{\left\{A_{n}\right\}}\right] \\
& \leq C_{1} \mathbb{E}\left[\bar{F}\left(\frac{u+\frac{\rho}{2} \mathcal{T}_{n}^{2}}{\mathcal{T}_{n}}\right)\right] \leq C_{1} \bar{F}\left(\sqrt{\frac{2 u}{\rho}}\right) .
\end{aligned}
$$

The theorem now follows from

$$
\sum_{n=g(u)}^{\epsilon \sqrt{u}} C_{1} \bar{F}\left(\sqrt{\frac{2 u}{\rho}}\right) \leq \epsilon C_{1} \sqrt{u} \bar{F}\left(\sqrt{\frac{2 u}{\rho}}\right)
$$

and $\epsilon \rightarrow 0$.

Corollary 5.2. If $X_{1}, X_{2}, \ldots$ are i.i.d. random variables with distribution $F(x)$ that is regularly varying with index $\alpha>1$, mean $\mu$, and regularly varying density $f(x)$. further let $a>0$ then the risk process

$$
R_{t}^{(1)}=u+p(t)-\sum_{i=1}^{N_{t}}\left(a+\mathcal{T}_{i}\right) X_{i} .
$$

has ruin probability

$$
\psi^{(1)}(u) \sim \psi(u)
$$

where $\psi(u)$. 
Proof. Since $R_{t}^{(1)} \leq R_{t}$ we get that $\psi^{(1)}(u) \gtrsim \psi(u)$. For the upper bound choose an $\epsilon>0$ and define the risk process

$$
R_{t}^{(\epsilon)}=u+p(t)-(1+\epsilon) \sum_{i=1}^{N_{t}} \mathcal{T}_{i} X_{i}
$$

If $\epsilon$ is small enough such that

$$
\rho_{\epsilon}=\rho-\epsilon \frac{\lambda \mu}{1+\epsilon}>0
$$

then by Theorem 5.1

$$
\psi^{(\epsilon)}(u) \sim \sqrt{u} \bar{F}\left(\frac{\sqrt{u}}{1+\epsilon}\right) \int_{0}^{\infty}\left(\frac{1}{t}+\frac{\rho_{\epsilon}}{2} t\right)^{-\alpha} \mathrm{d} t \leq(1+o(\epsilon)) \psi(u) .
$$

Denote with $t_{\epsilon}=a / \epsilon$ then by a coupling argument

$$
\begin{aligned}
\psi^{(1)}(u) & \leq \mathbb{P}\left(\inf _{0<t<t_{\epsilon}} R_{t}^{(1)}<u-\sqrt{u}\right)+\mathbb{P}\left(\inf _{t_{\epsilon}<t}\left(R_{t}^{(1)}-R_{t_{\epsilon}}^{(1)}\right)<\sqrt{u}-u\right) \\
& \leq \mathbb{P}\left(\left(a+t_{\epsilon}\right) \sum_{i=1}^{N_{t_{\epsilon}}} X_{i}>\sqrt{u}\right)+\mathbb{P}\left(\inf _{t_{\epsilon}<t}\left(R_{t}^{(\epsilon)}-R_{t_{\epsilon}}^{(\epsilon)}\right)<\sqrt{u}-u\right) \\
& \lesssim \lambda t_{\epsilon}\left(a+t_{\epsilon}\right)^{-\alpha} \bar{F}(\sqrt{u})+\psi^{(\epsilon)}\left(u-\sqrt{u}-p\left(t_{\epsilon}\right)\right)=(1+o(\epsilon)) \psi(u) .
\end{aligned}
$$

The corollary follows with $\epsilon \rightarrow 0$.

Lemma 5.3. Let $X_{1}, X_{2}, \ldots$ be regularly varying and $\mathcal{T}_{i}$ the time of the $i$-th claim. Assume that $p(t) \sim \frac{\mu+\rho}{2} \lambda t^{2}$ the collected premium up to time $t$. Further let $k>3, \epsilon<1 / k^{2}$ such that

$$
\frac{k-3.5}{k}>\left(1+1 / k^{2}\right) \frac{\mu+\rho / 2}{\mu+\rho} \text { and } \frac{4 \epsilon}{1-\epsilon} \frac{\mu}{\rho}<1 .
$$

Assume

$$
\left|\sum_{i=1}^{m} \mathcal{T}_{i}-\frac{m^{2}}{2 \lambda}\right| \leq \epsilon \frac{m^{2}}{2 \lambda}
$$

for $m \in\left(n, n-1, m_{n}\right)$, where $m_{n}=\lfloor(1-k \epsilon) n\rfloor,\left|\mathcal{T}_{n}-n / \lambda\right| \leq \epsilon n / \lambda$ and $\left|\mathcal{T}_{m_{n}}-m_{n} / \lambda\right| \leq$ $\epsilon m_{n} / \lambda$ then there exists a constant $C_{1}$ and $u_{0}$ such that for all $u>u_{0}, n>\log (\bar{F}(u))^{2}$ and $\mathcal{T}_{i} \leq \mathcal{T}_{n}$ which fulfill (4) we have that

$$
\begin{aligned}
& \mathbb{P}\left(\sum_{i=1}^{n} \frac{\mathcal{T}_{i}}{\mathcal{T}_{n}} X_{i}>\frac{u+p\left(\mathcal{T}_{n}\right)}{\mathcal{T}_{n}}, \sum_{i=1}^{n-1} \frac{\mathcal{T}_{i}}{\mathcal{T}_{n}} X_{i} \leq \frac{u+p\left(\mathcal{T}_{n}\right)}{\mathcal{T}_{n}}\right) \\
& \leq C_{1} \mathbb{P}\left(X_{n}>\frac{u+\frac{\rho \lambda}{2} \mathcal{T}_{n}^{2}}{\mathcal{T}_{n}}\right) .
\end{aligned}
$$

Further for every $c_{1}>0$ there exists a function $o(\epsilon) \rightarrow 0$ as $\epsilon \rightarrow 0$ and an $u_{1}$ such that for all $u>u_{1}, n>c_{1}$ sqrtu and $\mathcal{T}_{i} \leq \mathcal{T}_{n}$ which fulfill (4) we have that

$$
\mathbb{P}\left(\sum_{i=1}^{n} \frac{\mathcal{T}_{i}}{\mathcal{T}_{n}} X_{i}>\frac{u+p\left(\mathcal{T}_{n}\right)}{\mathcal{T}_{n}}, \sum_{i=1}^{m_{1}} \mathcal{T}_{i} X_{i} \leq u+p\left(\mathcal{T}_{m_{1}}\right), \sum_{i=1}^{n-1} \frac{\mathcal{T}_{i}}{\mathcal{T}_{n}} X_{i} \leq \frac{u+p\left(\mathcal{T}_{n}\right)}{\mathcal{T}_{n}}\right)
$$




$$
\leq(1+o(\epsilon)) \mathbb{P}\left(X_{n}>\frac{u+\frac{\rho \lambda}{2} \mathcal{T}_{n}^{2}}{\mathcal{T}_{n}}\right) .
$$

Proof. We only prove (6) since (5) can be proved along the same line. Denote with

$$
\begin{aligned}
& B_{1}=\left\{\sum_{i=1}^{m_{1}} \mathcal{T}_{i} X_{i} \leq u+p\left(\mathcal{T}_{m_{1}}\right)\right\}, \\
& B_{2}=\left\{\sum_{i=1}^{n-1} \frac{\mathcal{T}_{i}}{\mathcal{T}_{n}} X_{i} \leq \frac{u+p\left(\mathcal{T}_{n}\right)}{\mathcal{T}_{n}}\right\} .
\end{aligned}
$$

Denote with $\delta_{1}=3 \epsilon$ and

$$
\delta_{2}=\min \left[\frac{\epsilon}{2}, \epsilon c_{1}^{2} \frac{\mu+\rho}{2 \lambda}\right]
$$

We have that

$$
\begin{aligned}
\mathbb{P}\left(\sum_{i=1}^{n} \frac{\mathcal{T}_{i}}{\mathcal{T}_{n}} X_{i}>\frac{u+p\left(\mathcal{T}_{n}\right)}{\mathcal{T}_{n}}, B_{1}, B_{2}\right) \\
=\mathbb{P}\left(\sum_{i=1}^{n} \frac{\mathcal{T}_{i}}{\mathcal{T}_{n}} X_{i}>\frac{u+p\left(\mathcal{T}_{n}\right)}{\mathcal{T}_{n}},\left|\frac{\sum_{i=1}^{n-1} \frac{\mathcal{T}_{i}}{\mathcal{T}_{n}} X_{i}}{\mu n / 2}-1\right| \leq \delta_{1}, B_{1}, B_{2}\right) \\
\quad+\mathbb{P}\left(\sum_{i=1}^{n} \frac{\mathcal{T}_{i}}{\mathcal{T}_{n}} X_{i}>\frac{u+p\left(\mathcal{T}_{n}\right)}{\mathcal{T}_{n}}, \frac{\sum_{i=1}^{n-1} \frac{\mathcal{T}_{i}}{\mathcal{T}_{n}} X_{i}}{\mu n / 2} \leq 1-\delta_{1}, B_{1}\right) \\
\quad+\mathbb{P}\left(\sum_{i=1}^{n} \frac{\mathcal{T}_{i}}{\mathcal{T}_{n}} X_{i}>\frac{u+p\left(\mathcal{T}_{n}\right)}{\mathcal{T}_{n}},\left(1+\delta_{1}\right) \mu n / 2<\sum_{i=1}^{n-1} \frac{\mathcal{T}_{i}}{\mathcal{T}_{n}} X_{i} \leq\left(1-\delta_{2}\right) \frac{u+p\left(\mathcal{T}_{n}\right)}{\mathcal{T}_{n}}, B_{1}\right) \\
\quad+\mathbb{P}\left(\sum_{i=1}^{n} \frac{\mathcal{T}_{i}}{\mathcal{T}_{n}} X_{i}>\frac{u+p\left(\mathcal{T}_{n}\right)}{\mathcal{T}_{n}},\left(1-\delta_{2}\right) \frac{u+p\left(\mathcal{T}_{n}\right)}{\mathcal{T}_{n}} \leq \sum_{i=1}^{n-1} \frac{\mathcal{T}_{i}}{\mathcal{T}_{n}} X_{i} \leq \frac{u+p\left(\mathcal{T}_{n}\right)}{\mathcal{T}_{n}}, B_{1}\right) \\
=I_{1}+I_{2}+I_{3}+I_{4}
\end{aligned}
$$

Denote with

$$
B_{3}=\left\{\left|\frac{\sum_{i=1}^{n-1} \frac{\mathcal{T}_{i}}{\mathcal{T}_{n}} X_{i}}{\mu n / 2}-1\right| \leq \delta_{1}\right\} .
$$

The sets $B_{1}, B_{2}$ and $B_{3}$ only depend on $X_{1}, \ldots, X_{n-1}$. On the set $B_{3}$ we have

$$
\sum_{i=1}^{n-1} \frac{\mathcal{T}_{i}}{\mathcal{T}_{n}} X_{i} \leq\left(1+\delta_{1}\right) \mu n / 2 \leq \frac{1+\delta_{1}}{1-\epsilon} \mu \lambda \frac{\mathcal{T}_{n}}{2}
$$

and hence

$$
\begin{aligned}
& \frac{u+p\left(\mathcal{T}_{n}\right)}{\mathcal{T}_{n}}-\sum_{i=1}^{n-1} \frac{\mathcal{T}_{i}}{\mathcal{T}_{n}} X_{i} \gtrsim \frac{u+\frac{\mu+\rho}{2} \lambda \mathcal{T}_{n}^{2}}{\mathcal{T}_{n}}-\frac{1+\delta_{1}}{1-\epsilon} \mu \lambda \frac{\mathcal{T}_{n}}{2} \\
& =\frac{u}{\mathcal{T}_{n}}+\left(1-\frac{4 \epsilon}{1-\epsilon} \frac{\mu}{\rho}\right) \frac{\rho \lambda \mathcal{T}_{n}}{2} \geq\left(1-\frac{4 \epsilon}{1-\epsilon} \frac{\mu}{\rho}\right) \frac{u+\frac{\lambda \rho}{2} \mathcal{T}_{n}^{2}}{\mathcal{T}_{n}}
\end{aligned}
$$


Since $X_{n}$ is regularly varying we get

$$
I_{1} \lesssim \mathbb{P}\left(X_{n}>\left(1-\frac{4 \epsilon}{1-\epsilon} \frac{\mu}{\rho}\right) \frac{u+\frac{\lambda \rho}{2} \mathcal{T}_{n}^{2}}{\mathcal{T}_{n}}\right) \sim\left(1-\frac{4 \epsilon}{1-\epsilon} \frac{\mu}{\rho}\right)^{-\alpha} \mathbb{P}\left(X_{n}>\frac{u+\frac{\lambda \rho}{2} \mathcal{T}_{n}^{2}}{\mathcal{T}_{n}}\right)
$$

$I_{2}$ is basically a large deviation probability. Note that there exists an $s_{\epsilon}$ such that for $0 \leq s \leq s_{\epsilon}-\log \left(\mathbb{E}\left[e^{-s X_{i}}\right]\right)>(1-\epsilon) s \mu$ and hence we get by Chebyshev inequality that

$$
\begin{aligned}
I_{2} & \leq \mathbb{P}\left(\frac{\sum_{i=1}^{n-1} \frac{\mathcal{T}_{i}}{\mathcal{T}_{n}} X_{i}}{\mu n / 2} \leq 1-\delta_{1}\right) \leq \exp \left(s_{\epsilon}\left(1-\delta_{1}\right) \mu \frac{n}{2}+\sum_{i=1}^{n-1} \log \left(\mathbb{E}\left[e^{-s_{\epsilon} \frac{\mathcal{T}_{i}}{\mathcal{T}_{n}} X_{i}}\right]\right)\right) \\
& \leq \exp \left(s_{\epsilon}\left(1-\delta_{1}\right) \mu \frac{n}{2}-(1-\epsilon) \sum_{i=1}^{n-1} s_{\epsilon} \frac{\mathcal{T}_{i}}{\mathcal{T}_{n}} \mu\right) \\
& \leq \exp \left(s_{\epsilon}\left(1-\delta_{1}\right) \mu \frac{n}{2}-\frac{(1-\epsilon)(1-\epsilon)}{1+\epsilon} s_{\epsilon} \frac{n}{2} \mu\right) \\
& =\exp \left(s_{\epsilon} \mu \frac{n}{2} \frac{\left(1-\delta_{1}\right)(1+\epsilon)-(1-\epsilon)(1-\epsilon)}{1+\epsilon}\right) \\
& =\exp \left(-s_{\epsilon} \mu \frac{n}{2} \frac{4 \epsilon^{2}}{1+\epsilon}\right)
\end{aligned}
$$

Hence for $n>\log (\bar{F}(u))^{2}$ it follows that

$$
I_{2}=o\left(\mathbb{P}\left(X_{n}>\frac{u+p\left(\mathcal{T}_{n}\right)}{\mathcal{T}_{n}}\right)\right)
$$

Ad $I_{3}$ : Note that

$$
\frac{\epsilon\left(1+\delta_{1}\right)(1-\epsilon)}{1+\epsilon}=\frac{(1+3 \epsilon)(1-\epsilon)}{1+\epsilon}>1 .
$$

By Lemma B.4 we get that

$$
\begin{aligned}
I_{3} & \leq \mathbb{P}\left(X_{n}>\delta_{2} \frac{u+p\left(\mathcal{T}_{n}\right)}{\mathcal{T}_{n}}, \sum_{i=1}^{n-1} \frac{\mathcal{T}_{i}}{\mathcal{T}_{n}} X_{i}>\left(1+\delta_{1}\right) \mu n / 2\right) \\
& =\mathbb{P}\left(X_{n}>\delta_{2} \frac{u+p\left(\mathcal{T}_{n}\right)}{\mathcal{T}_{n}}\right) \mathbb{P}\left(\sum_{i=1}^{n-1} \frac{\mathcal{T}_{i}}{\mathcal{T}_{n}} X_{i}>\left(1+\delta_{1}\right) \mu n / 2\right) \\
& \leq \mathbb{P}\left(X_{n}>\delta_{2} \frac{u+p\left(\mathcal{T}_{n}\right)}{\mathcal{T}_{n}}\right) \mathbb{P}\left(\sum_{i=1}^{n-1} \frac{\mathcal{T}_{i}}{\mathcal{T}_{n}} X_{i}>\frac{\left(1+\delta_{1}\right)(1-\epsilon)}{1+\epsilon} \mu \sum_{i=1}^{n-1} \frac{\mathcal{T}_{i}}{\mathcal{T}_{n}}\right) \\
& =o\left(\mathbb{P}\left(X_{n}>\frac{u+p\left(\mathcal{T}_{n}\right)}{\mathcal{T}_{n}}\right)\right) .
\end{aligned}
$$

Finally we are left with $I_{4}$. We want to apply Lemma A.3. When $\sum_{i=1}^{m_{n}} \mathcal{T}_{i} X_{i} \leq u+p\left(\mathcal{T}_{m_{n}}\right)$ and $\sum_{i=1}^{n} \frac{\mathcal{T}_{i}}{\mathcal{T}_{n-1}} X_{i}>\left(1-\delta_{2}\right) \frac{u+p\left(\mathcal{T}_{n}\right)}{\mathcal{T}_{n}}$ then for

$$
\gamma=\frac{\epsilon}{2}
$$


there exists an $u_{0}$ such that for all $u>u_{0}$ and $n>c_{1} \sqrt{u}$

$$
\begin{aligned}
\sum_{i=m_{n}+1}^{n} \frac{\mathcal{T}_{i}}{\mathcal{T}_{n}} X_{i} & >\left(1-\delta_{2}\right) \frac{u+p\left(\mathcal{T}_{n}\right)}{\mathcal{T}_{n}}-\frac{u+p\left(\mathcal{T}_{m_{n}}\right)}{\mathcal{T}_{n}} \\
& >\left(1-\delta_{2}\right) \frac{u+(1-\gamma) \frac{\mu+\rho}{2} \lambda \mathcal{T}_{n}^{2}}{\mathcal{T}_{n}}-\frac{u+(1+\gamma) \frac{\mu+\rho}{2} \lambda \mathcal{T}_{m_{1}}^{2}}{\mathcal{T}_{n}} \\
& >\left(1-\delta_{2}\right) \frac{(1-\gamma)(1-\epsilon) \frac{\mu+\rho}{2 \lambda} n^{2}}{\mathcal{T}_{n}}-\frac{\delta_{2} c_{1}^{-2} n^{2}+(1+\gamma)(1+\epsilon) \frac{\mu+\rho}{2 \lambda}(1-k \epsilon)^{2} n^{2}}{\mathcal{T}_{n}} \\
& \geq \frac{(\mu+\rho) n^{2}}{2 \lambda \mathcal{T}_{n}}\left(1-\epsilon-(1+\epsilon)(1-k \epsilon)^{2}-3 \epsilon\right) \quad \text { definition of } \delta_{2}, \gamma \\
& =\frac{(\mu+\rho) n^{2}}{2 \lambda \mathcal{T}_{n}}\left((2 k-5) \epsilon+\left(2 k-k^{2}\right) \epsilon^{2}-k^{2} \epsilon^{3}\right) \\
& \geq \frac{(\mu+\rho) n^{2}}{2 \lambda \mathcal{T}_{n}}(2 k-7) \epsilon \quad \epsilon<1 /\left(k^{2}\right) \\
& \geq \frac{(\mu+\rho)}{(1+\epsilon)}\left(n-m_{n}\right) \frac{(k-3.5) n \epsilon}{n-m_{n}} \\
& \geq \frac{(\mu+\rho)}{(1+\epsilon)}\left(n-m_{n}\right) \frac{\epsilon(k-3.5) n}{\epsilon k n+1} .
\end{aligned}
$$

From the condition on $k$ we get that for $n$ large enough

$$
\sum_{i=m_{n}+1}^{n} \frac{\mathcal{T}_{i}}{\mathcal{T}_{n}} X_{i} \geq(\mu+\rho / 2)\left(n-m_{n}\right) \geq(\mu+\rho / 2) \sum_{i=m_{1}+1}^{n} \frac{\mathcal{T}_{i}}{\mathcal{T}_{n}} .
$$

From Lemma A.3 we get that for $\left(1-\delta_{2}\right) \frac{u+p\left(\mathcal{T}_{n}\right)}{\mathcal{T}_{n}} \leq x<\frac{u+p\left(\mathcal{T}_{n}\right)}{\mathcal{T}_{n}}$ some $C_{1}>0$ and $u$ large enough that

$$
\begin{aligned}
& \mathbb{P}\left(\sum_{i=m_{n}+1}^{n} \frac{\mathcal{T}_{i}}{\mathcal{T}_{n}} X_{i}>(\mu+\rho / 2) \sum_{i=m_{1}+1}^{n} \frac{\mathcal{T}_{i}}{\mathcal{T}_{n}}, \sum_{i=1}^{n-1}, \frac{\mathcal{T}_{i}}{\mathcal{T}_{n}} \in[x, x+1)\right) \\
& \leq C_{1} k \in n \mathbb{P}\left(X_{1} \in[x, x+1)\right)
\end{aligned}
$$

and Lemma B.3 we get that for some $C_{2}>0$ (that can depend on $k$ )

$$
\begin{aligned}
& I_{4} \leq \mathbb{P}\left(\left(1-\delta_{2}\right) \frac{u+p\left(\mathcal{T}_{n}\right)}{\mathcal{T}_{n}} \leq \sum_{i=1}^{n-1} \frac{\mathcal{T}_{i}}{\mathcal{T}_{n}} X_{i} \leq \frac{u+p\left(\mathcal{T}_{n}\right)}{\mathcal{T}_{n}}\right. \\
& \left.\sum_{i=1}^{n} \frac{\mathcal{T}_{i}}{\mathcal{T}_{n}} X_{i}>\frac{u+p\left(\mathcal{T}_{n}\right)}{\mathcal{T}_{n}}, \sum_{m_{n}+1}^{n} \frac{\mathcal{T}_{i}}{\mathcal{T}_{n}} X_{i}>(\mu+\rho / 2) \sum_{i=m_{1}+1}^{n} \frac{\mathcal{T}_{i}}{\mathcal{T}_{n}}\right) \\
& \leq \epsilon C_{2} n f\left(\frac{u+p\left(\mathcal{T}_{n}\right)}{\mathcal{T}_{n}}\right) \\
& \sim \epsilon C_{2} n\left(\frac{u+p\left(\mathcal{T}_{n}\right)}{\mathcal{T}_{n}}\right)^{-1} \bar{F}\left(\frac{u+p\left(\mathcal{T}_{n}\right)}{\mathcal{T}_{n}}\right)
\end{aligned}
$$


where the last line follows from $f$ regularly varying. The Lemma follows since

$$
n\left(\frac{u+p\left(\mathcal{T}_{n}\right)}{\mathcal{T}_{n}}\right)^{-1}
$$

is bounded.

\section{$6 \quad$ Numerical examples}

The speed of future climate change and global warming remains uncertain and specialists use numerical models (most of the time general circulation models) representing physical processes in the atmosphere, ocean and land surface. The main parameter in these models is the human emissions of greenhouse gases, mainly $\mathrm{CO}_{2}$. The Intergovernmental Panel on Climate Change (IPCC) provides different emission scenarios families (namely A1, A2, B1 and B2) corresponding to different sets of hypotheses about economic growth, population evolution, certain gas emissions, mitigation strategies, and many other factors (see [12]). Usually, the B1 scenario is considered the most optimistic one while A1FI is the pessimistic one.

It would be very challenging to quantify the impact of climate change on the claim size distribution of some insurer for each scenario. Here for illustration we consider different impacts on the claim size distribution, described by different values of parameter $c_{\alpha}$ that controls the claim size worsening speed. We take $u=500, c_{\alpha} \in\{1,2,5,10,20\} \%, \alpha_{0}=1.5$, $d_{0}=0$ and $\rho=1$ or 0.1 . Furthermore, we only calculate the ruin problem up to time $T$ where $T$ is the first time that $\mu(t)$ (the premium income rate at time $t$ ) exceeds the maximal acceptable premium intensity $\mu$-max, that is chosen such that for $c=0.1$ we have $T=20$. We also compute the probability of ruin up to time $T=1000$ and give asymptotic estimates for the infinite time ruin problem. We also give some plots of sample paths of the considered process or of rescaled versions of the considered processes in Figures 1-4. Tables 3 and 4 are made in a different way. We first calculate (for every $c_{\alpha}$ ) $\mu$-max such that for $\rho=1, \mu$-max is 20 years (results in Table 3), then we use the the same $\mu$-max for $\rho=0.1$ (results in 4), in order to see what happens when the safety loading is smaller. As one could expect, it is then longer to reach $\mu$-max in that case, and it is more likely to be ruined before reaching this time horizon than in the previous case (when the safety loading was $100 \%$ ). In both cases, one can see that being ruined before this time horizon is often neither neglectable nor close to 1: it is thus interesting to study it. We left some inconsistent asymptotic results (including values $>1$ in Tables 2 and 4) to illustrate that for some parameters, it is clear that we are no longer in the asymptotic theory application domain. For the second model with parameter $\rho=0.1$ we also calculated estimates for the ruin probability before $T=10^{5}$ with 100 simulations; in this case all estimates were bigger than 0.75 , so for these sets of parameters we do clearly not expect asymptotic results to hold. In general in the proposed models, it is not easy to determine whether the asymptotic approximation is accurate or not, because we are dealing with infinite time ruin probabilities, with limited knowledge on the distribution of the time to ruin. We used $10^{7}$ simulations so the error (half-length $95 \%$-confidence interval) is approximated by $2 \sqrt{z / 10^{7}}$ where $z$ is the result. For $z=.002$ this gives for example an 


\begin{tabular}{|c|c||c|c||c|c|c|c|}
\hline$c$ & $T$ & ruin1 & ruin2 & as1 & $\mathrm{T}=1000$ & as2 & $\mathrm{T}=1000$ \\
\hline \hline 0.01 & 200 & 0.0401 & 0.0242 & 0.497 & 0.107 & 0.124 & 0.0672 \\
\hline 0.02 & 100 & 0.027 & 0.0166 & 0.351 & 0.105 & 0.147 & 0.087 \\
\hline 0.05 & 40 & 0.0134 & 0.00833 & 0.222 & 0.0918 & 0.185 & 0.119 \\
\hline 0.1 & 20 & 0.0073 & 0.00491 & 0.157 & 0.0784 & 0.22 & 0.146 \\
\hline 0.2 & 10 & 0.00422 & 0.00217 & 0.111 & 0.0643 & 0.262 & 0.179 \\
\hline
\end{tabular}

Table 1: Table of finite time ruin probabilities for different time horizon and claim size worsening speed together with the asymptotic for ruin probability for $\rho=1$.

error of approximately 0.000028 (for $T=1000$ the computation time is much longer and hence we only used $10^{5}$ simulations, which multiplies by 10 the above confidence interval half-length). The numbers in Tables 1 to 4 illustrate the tradeoff between risk of ruin and risk of inassurability: if one decreases the safety loading from 1 to 0.1 , then it becomes possible to propose acceptable prices during a longer period, but of course this causes a strong increase in the probability to be ruined before premium becomes unacceptably high. They also illustrate the potential impact of climate change or of some kinds of sectorial inflation on the long term profitability and solvency of some insurance businesses.

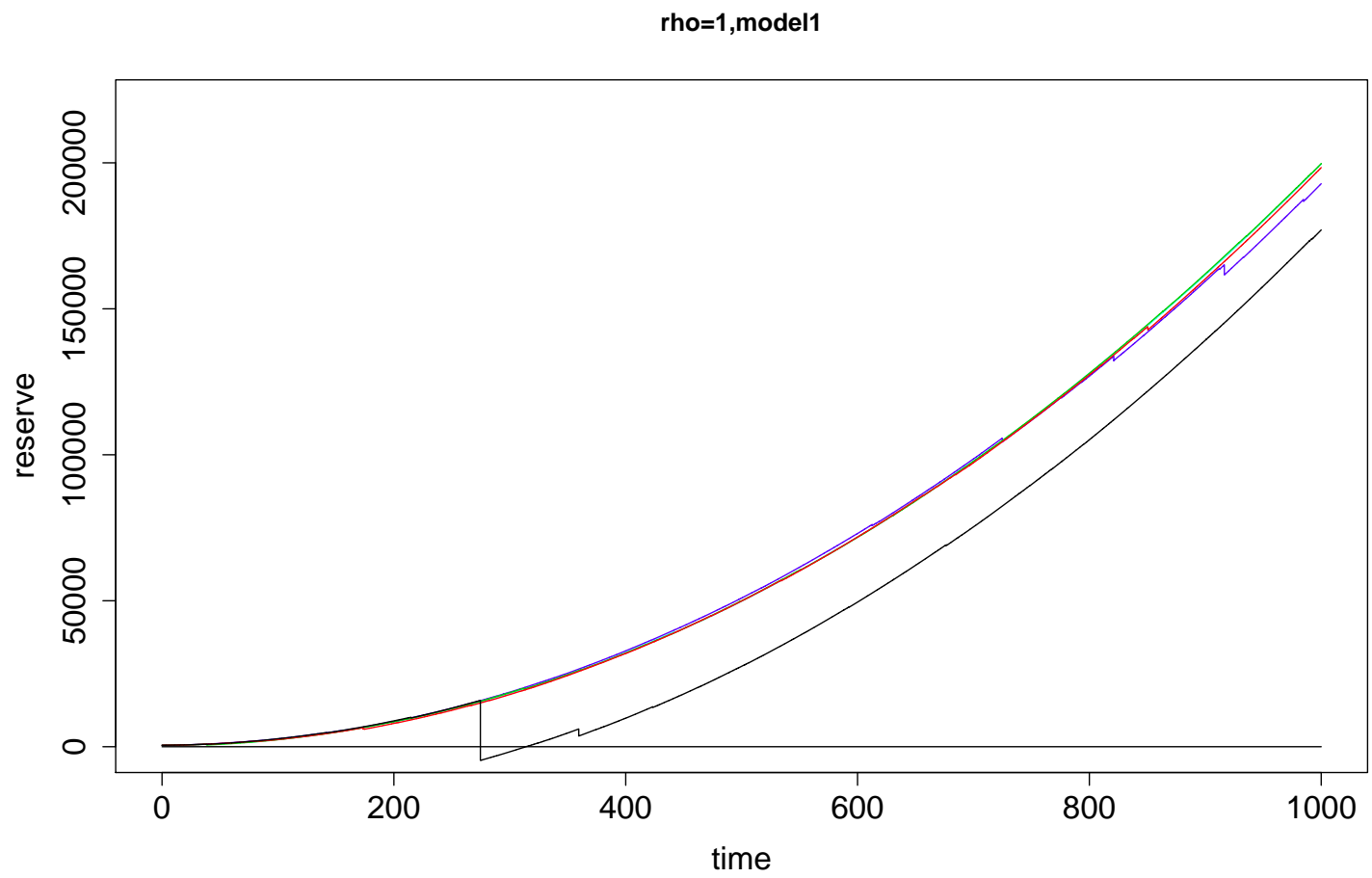

Figure 1: Plot of the surplus process for Model 1 with $\rho=1$ and $c=0.1$.

\section{Acknowlegments}

This work is mainly funded by the Research Chair Actuariat Responsable sponsored by Generali, and also supported by the Research Chair Actuariat Durable sponsored by Mil- 


\begin{tabular}{|c|c||c|c||c|c|c|c|}
\hline$c$ & $T$ & ruin1 & ruin2 & as1 & $\mathrm{T}=1000$ & as2 & $\mathrm{T}=1000$ \\
\hline \hline 0.01 & 200 & 0.069 & 0.0512 & 0.67 & 0.218 & 2.2 & 0.343 \\
\hline 0.02 & 100 & 0.0381 & 0.0272 & 0.474 & 0.197 & 2.62 & 0.443 \\
\hline 0.05 & 40 & 0.0164 & 0.0103 & 0.3 & 0.159 & 3.3 & 0.569 \\
\hline 0.1 & 20 & 0.00804 & 0.00573 & 0.212 & 0.129 & 3.92 & 0.646 \\
\hline 0.2 & 10 & 0.00424 & 0.00255 & 0.15 & 0.101 & 4.66 & 0.716 \\
\hline
\end{tabular}

Table 2: Table of finite time ruin probabilities for different time horizon and claim size worsening speed together with the asymptotic for ruin probability for $\rho=0.1$.

\begin{tabular}{|c|c||c||c|c||c|c|}
\hline$c$ & $\mu-\max$ & $\mathrm{T}$ & ruin1 & as1 & ruin2 & as2 \\
\hline \hline 0.01 & 4.8 & 20 & 0.00225 & 0.497 & 0.00197 & 0.124 \\
\hline 0.02 & 5.6 & 20 & 0.00287 & 0.351 & 0.00225 & 0.147 \\
\hline 0.05 & 8 & 20 & 0.00475 & 0.222 & 0.00312 & 0.185 \\
\hline 0.1 & 12 & 20 & 0.00748 & 0.157 & 0.00471 & 0.22 \\
\hline 0.2 & 20 & 20 & 0.0114 & 0.111 & 0.00838 & 0.262 \\
\hline
\end{tabular}

Table 3: Table of finite time ruin probabilities for time horizon 20 and different claim size worsening speed together with the asymptotic for ruin probability for $\rho=1$.

\begin{tabular}{|c|c||c||c|c||c|c|}
\hline$c$ & $\mu-\max$ & $\mathrm{T}$ & ruin1 & as1 & ruin2 & as2 \\
\hline \hline 0.01 & 4.8 & 118 & 0.0323 & 0.67 & 0.0223 & 2.2 \\
\hline 0.02 & 5.6 & 77.3 & 0.0256 & 0.474 & 0.0172 & 2.62 \\
\hline 0.05 & 8 & 52.7 & 0.0251 & 0.3 & 0.018 & 3.3 \\
\hline 0.1 & 12 & 44.5 & 0.0284 & 0.212 & 0.0253 & 3.92 \\
\hline 0.2 & 20 & 40.5 & 0.0326 & 0.15 & 0.0445 & 4.66 \\
\hline
\end{tabular}

Table 4: Table of finite time ruin probabilities for time horizon 20 and different claim size worsening speed together with the asymptotic for ruin probability for $\rho=1$. 


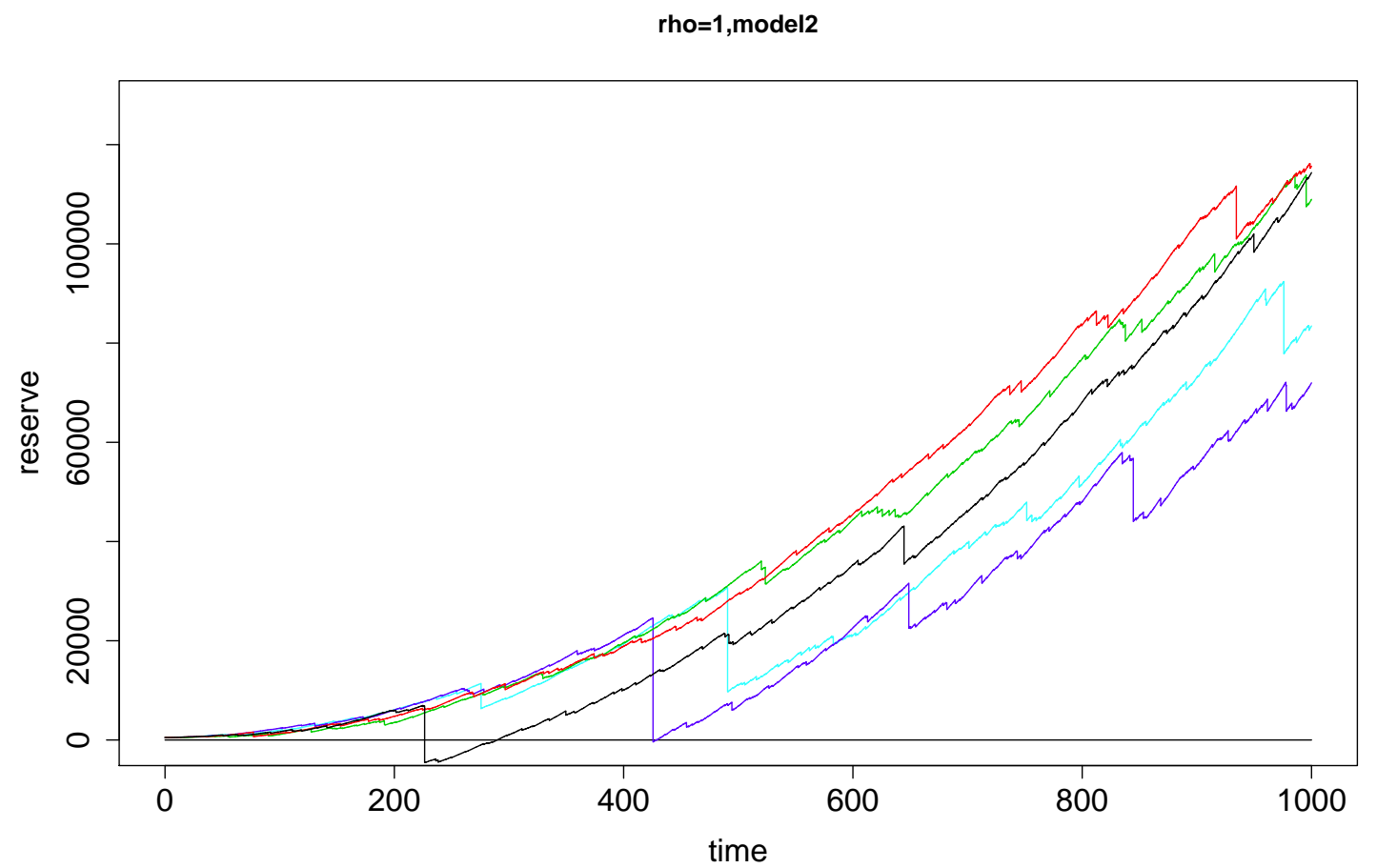

Figure 2: Plot of the surplus process for Model 2 with $\rho=1$ and $c=0.1$.

liman, and the MIRACCLE project sponsored by French ministry of ecology.

\section{A Large deviation auxiliary results}

To prove an asymptotic upper bound we need large deviation results for heavy tailed random variables a recent paper on this topic is [9] where a quite general large deviation result is shown. An application of $[9$, Theorem 2.1] yields

Corollary A.1. If $X_{1}, X_{2}, \ldots$ are i.i.d. random variables with distribution $F(x)$ that is regularly varying with index $0<\alpha \leq 1$, and regularly varying density $f(x)$. If further $p(T)$ is regularly varying with index $\beta>1 / \alpha$ then for all (fixed) $\epsilon>0$ and $T>0$

$$
\lim _{n \rightarrow \infty} \sup _{x \geq \epsilon p(n / \lambda)}\left|\frac{\mathbb{P}\left(S_{n} \in(x, x+T]\right)}{n(F(x)-F(x+T))}-1\right|=0 .
$$

The result also holds for $T=\infty$.

Proof. We should mention that this result is quite close to the examples in [9] so we can basically copy their proves with obvious modifications. To meet the assumptions of their Theorem 2.1 we need four series $b_{n}, h_{n}, I_{n}$ and $J_{n}$ such that for ever $\epsilon>0$ there exits an $K$ such that for all $n$

$$
\mathbb{P}\left(S_{n} / b_{n} \leq K\right)>1-\epsilon
$$




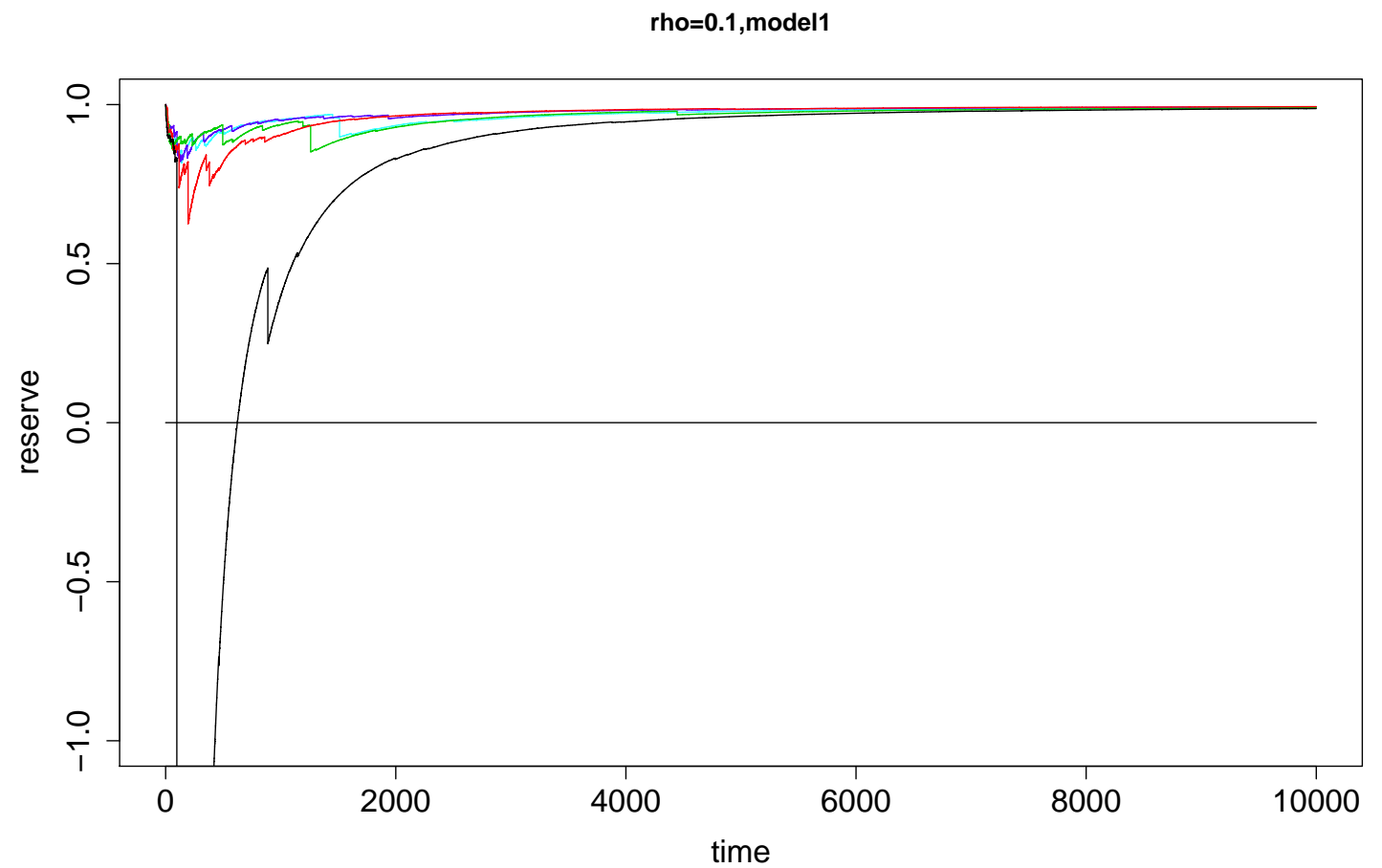

Figure 3: Plot of the rescaled surplus process (surplus process divided by $(u+p(t))$ ) for Model 1 with $\rho=0.1$ and $c=0.1$.

Further we need that $b_{n} / I_{n} \rightarrow 0$ and that for every $T>0$

$$
\lim _{n \rightarrow \infty} \sup _{x \geq I_{n}} \sup _{0 \leq t \leq b_{n}}\left|\frac{F(x-t+T)-F(x-t)}{F(x+T)-F(x)}-1\right|=0
$$

The series $h_{n}$ has to fulfill $h_{n}=\mathcal{O}\left(b_{n}\right)$ and for all $T>0$.

$$
\lim _{n \rightarrow \infty} \sup _{x \geq h_{n}} \frac{n \mathbb{P}\left(X_{1}+X_{2} \in(x, x+T], \min \left(X_{1}, X_{2}\right)>h_{n}\right)}{F(x+T)-F(x)}=0 .
$$

and finally the sequence $J_{n} \geq h_{n}$ fulfills

$$
\lim _{n \rightarrow \infty} \sup _{x \geq J_{n}} \sup _{z \geq x} \frac{\mathbb{P}\left(S_{n} \in(x, x+T], X_{1} \leq h_{n}, \ldots, X_{n} \leq h_{n}\right)}{n(F(x)-F(x+T))} .
$$

Now to prove the corollary it is enough to show that for every $\beta_{2}>\beta_{1}>1 / \alpha$ we can choose $h_{n}=b_{n}=n^{\beta_{1}}$, and $I_{n}=J_{n}=n^{\beta_{2}}$.

As in $[9]$ we get from $\mathbb{E}\left[X^{1 / \beta_{1}}\right]<\infty$ by the Marcinkiewicz-Zygmund law of large numbers (e.g. [10, Theorem 2.1.5]) that $b_{n}=n^{\beta_{1}}$ fulfills the required condition. By a Taylor argument we get for $0 \leq \xi_{i} \leq T$

$$
\sup _{x \geq n^{\beta_{2}}} \sup _{0 \leq t \leq n^{\beta_{1}}}\left|\frac{F(x-t+T)-F(x-t)}{F(x+T)-F(x)}-1\right|=\sup _{x \geq n^{\beta_{2}}} \sup _{0 \leq t \leq n^{\beta_{1}}}\left|\frac{f\left(x-t+\xi_{1}\right)}{f\left(x+\xi_{2}\right)}-1\right|
$$




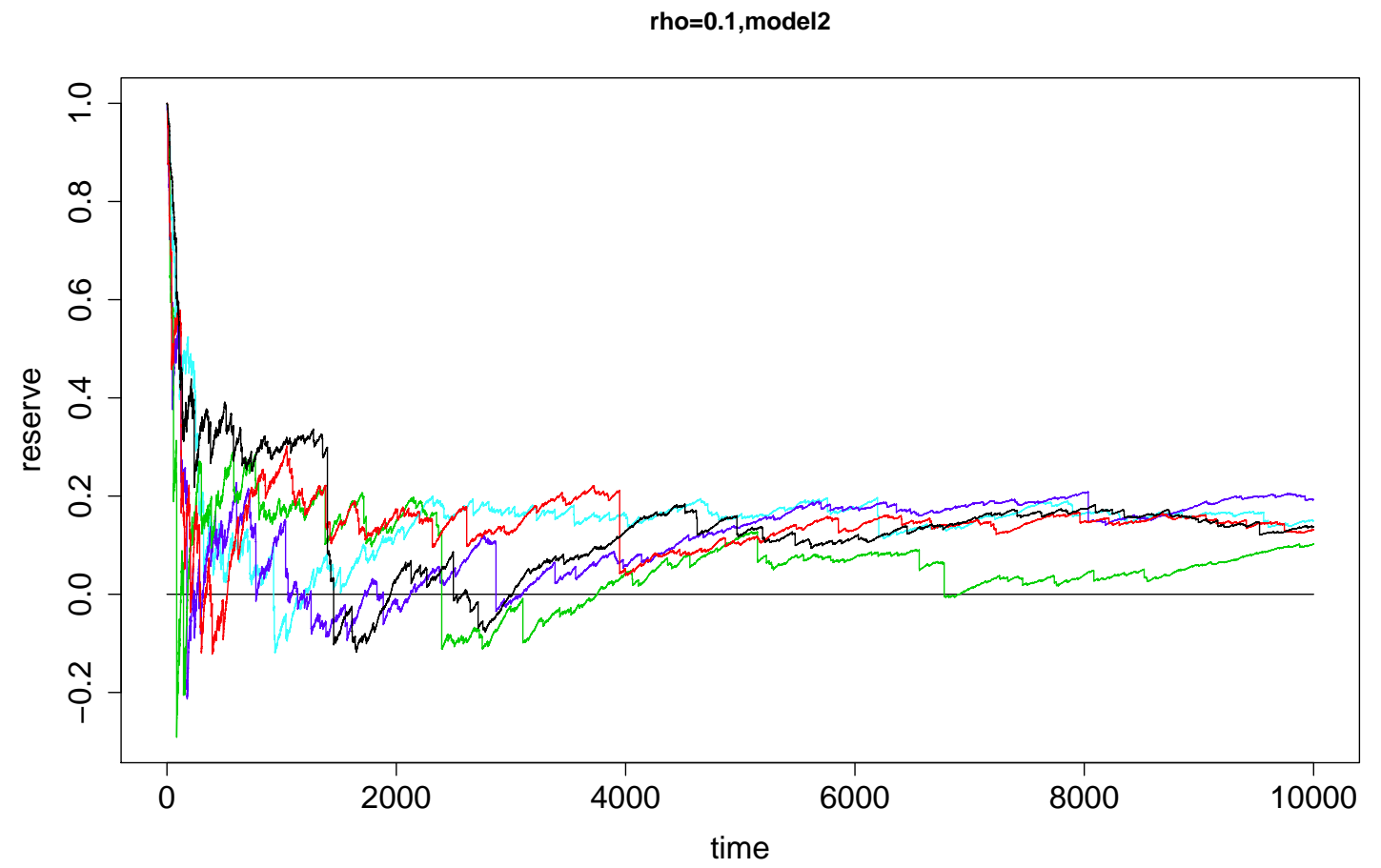

Figure 4: Plot of the rescaled surplus process (surplus process divided by $(u+p(t)))$ for Model 2 with $\rho=0.1$ and $c=0.1$.

$$
\lesssim \sup _{x \geq n^{\beta_{2}}} \sup _{0 \leq t \leq n^{\beta_{1}}}\left|\frac{f\left(x-n^{\beta_{1}}\right)}{f(x+T)}-1\right| \rightarrow 0
$$

since $n^{\beta_{1}-\beta_{2}} \rightarrow 0$.

Note that for $x>2 h_{n}-T$

$$
\begin{aligned}
& \mathbb{P}\left(X_{1}+X_{2} \in(x, x+T], \min \left(X_{1}, X_{2}\right)>h_{n}\right) \\
& \leq \int_{h_{n}}^{x+T-h_{n}} \int_{(x-y) \vee h_{n}}^{x+T-y} f(z) f(y) \mathrm{d} x \mathrm{~d} y \lesssim T \int_{h_{n}}^{x+T-h_{n}} f(y) f\left((x-y) \vee h_{n}\right) \mathrm{d} x \mathrm{~d} y \\
& \lesssim T \int_{h_{n}}^{x / 2}+\int_{x / 2}^{x+T-h_{n}} f(y) f(x-y) \mathrm{d} x \mathrm{~d} y \lesssim T f(x / 2) \bar{F}\left(h_{n}\right)+T f(x / 2) \bar{F}\left(h_{n}-T\right) .
\end{aligned}
$$

Since $n \bar{F}\left(h_{n}-T\right) \rightarrow 0$ it follows

$$
\lim _{n \rightarrow \infty} \sup _{x \geq h} \frac{n \mathbb{P}\left(X_{1}+X_{2} \in(x, x+T], \min \left(X_{1}, X_{2}\right)>h_{n}\right)}{F(x+T)-F(x)}
$$

Finally we have to show that

$$
\lim _{n \rightarrow \infty} \sup _{x \geq J_{n}} \sup _{z \geq x} \frac{\mathbb{P}\left(S_{n} \in(x, x+T], X_{1} \leq h_{n}, \ldots, X_{n} \leq h_{n}\right)}{n(F(x)-F(x+T))}=0 .
$$


Therefore note that $\left(e^{x} \leq 1+2 x 0 \leq x \leq 1\right)$

$$
\begin{aligned}
& \mathbb{P}\left(S_{n} \in(x, x+T], X_{1} \leq h_{n}, \ldots, X_{n} \leq h_{n}\right) \\
& =\int_{\left[0, h_{n}\right]^{n}} f\left(x_{1}\right) \cdots f\left(x_{n}\right) 1_{\left\{S_{n} \in(x, x+T]\right\}} \mathrm{d} x_{1} \ldots \mathrm{d} x_{n} \\
& =\int_{\left[0, h_{n}\right]^{n}} e^{-S_{n} / h_{n}} \prod_{k=1}^{n}\left(e^{x_{k} / h_{n}} f\left(x_{k}\right)\right) 1_{\left\{S_{n} \in(x, x+T]\right\}} \mathrm{d} x_{1} \ldots \mathrm{d} x_{n} \\
& \leq e^{-x / h_{n}} \int_{\left[0, h_{n}\right]^{n}} \prod_{k=1}^{n}\left(e^{x_{k} / h_{n}} f\left(x_{k}\right)\right) \mathrm{d} x_{1} \ldots \mathrm{d} x_{n} \\
& =e^{-x / h_{n}}\left(\int_{0}^{h_{n}} e^{y / h_{n}} f(y) \mathrm{d} y\right)^{n} \\
& =\exp \left[-x / h_{n}+n \log \left(1+2 \int_{0}^{h_{n}} \frac{y}{h_{n}} f(y) \mathrm{d} y\right)\right] .
\end{aligned}
$$

Since

$$
n \log \left(1+2 \int_{0}^{h_{n}} \frac{y}{h_{n}} f(y) \mathrm{d} y\right) \sim \frac{2 n \int_{0}^{h_{n}} y f(y) \mathrm{d} y}{h_{n}}
$$

is regularly varying with index $1-\beta_{1}+(1-\alpha) \beta_{1}=1-\alpha \beta_{1}<0$ it follows

$$
\mathbb{P}\left(S_{n} \in(x, x+T], X_{1} \leq h_{n}, \ldots, X_{n} \leq h_{n}\right) \leq C e^{-x / h_{n}}=o(f(x)) .
$$

We also need a large deviation result for non identically distributed random variables. Nevertheless one should note that the method of [9] still works and we only have to make the obvious modifications. We first give an auxiliary result that is related to the small step sequences $h_{n}$ and $J_{n}$ of the proof of Corollary A.1.

Lemma A.2. Let $X_{1}, X_{2}, \ldots$ be regularly varying with regularly varying density $f$ and index $\alpha>1$. Denote with $\mu=\mathbb{E}\left[X_{1}\right]$. Then for every fixed $0<\gamma<1, c_{1}, c_{2}, a>0$

$$
\lim _{n \rightarrow \infty} \sup _{\mathcal{T}_{i} \leq \mathcal{T}_{n}} \sup _{x>\frac{1}{a}\left(c_{1} n \vee\left(1+c_{2}\right) \mu \sum_{i=1}^{n-1} \frac{\mathcal{T}_{i}}{\mathcal{T}_{n}}\right)} \sup _{c_{3}>a} \frac{\mathbb{P}\left(\sum_{i=1}^{n-1} \frac{\mathcal{T}_{i}}{\mathcal{T}_{n}} X_{i}>c_{3} x, \frac{\mathcal{T}_{i}}{\mathcal{T}_{n}} X_{i} \leq x^{\gamma}\right)}{n f(x)}=0 .
$$

Proof. W.l.o.g. we can use $c_{3}=a$. Then

$$
\begin{aligned}
& \mathbb{P}\left(\sum_{i=1}^{n-1} \frac{\mathcal{T}_{i}}{\mathcal{T}_{n}} X_{i}>a x, \frac{\mathcal{T}_{i}}{\mathcal{T}_{n}} X_{i} \leq x^{\gamma}\right) \\
& =\int_{\prod_{i=1}^{n-1}\left[0, \frac{\mathcal{T}_{n}}{\mathcal{T}_{i}} x^{\gamma}\right]} e^{-\frac{1}{x^{\gamma}} \sum_{i=1}^{n-1} \frac{\mathcal{T}_{i}}{\mathcal{T}_{n}} x_{i}} 1_{\left\{\sum_{i=1}^{n-1} \frac{\mathcal{T}_{i}}{\mathcal{T}_{n}} x_{i}>a x\right\}} \prod_{i=1}^{n-1} e^{\frac{\mathcal{T}_{i}}{\mathcal{T}_{n} x^{\prime}} x_{i}} f\left(x_{i}\right) \mathrm{d} \mathbf{x} \\
& \leq \exp \left(-\frac{a x}{x^{\gamma}}+\sum_{i=1}^{n-1} \log \left(\int_{0}^{\frac{\mathcal{T}_{n}}{\mathcal{T}_{i}} x^{\gamma}} e^{\frac{\mathcal{T}_{i}}{\mathcal{T}_{n} x} y} f(y) \mathrm{d} y\right)\right)
\end{aligned}
$$


For $\delta<\alpha-1$ and constants $C_{i}>0$ we get that there exists a $d>1$ such that $f(x) \leq$ $C_{1} y^{-\alpha-1+\delta}$ for all $x>d$. Since $e^{x}-1-x \leq x\left(e^{d}-(d+1)\right)$ for $0<x<d$ we get that

$$
\begin{aligned}
& \int_{0}^{\frac{\mathcal{T}_{n}}{\mathcal{T}_{i}} x^{\gamma}} e^{\frac{\mathcal{T}_{i}}{\mathcal{T}_{n} x^{\gamma}} y} f(y) \mathrm{d} y=\sum_{k=0}^{\infty}\left(\frac{\mathcal{T}_{i}}{\mathcal{T}_{n} x^{\gamma}}\right)^{k} \frac{1}{k !} \int_{0}^{\frac{\mathcal{T}_{n}}{\mathcal{T}_{i}} x^{\gamma}} y^{k} f(y) \mathrm{d} x \\
& \leq 1+\frac{\mu}{x^{\gamma}} \frac{\mathcal{T}_{i}}{\mathcal{T}_{n}}+\sum_{k=2}^{\infty}\left(\frac{\mathcal{T}_{i}}{\mathcal{T}_{n} x^{\gamma}}\right)^{k} \frac{d^{k}}{k !}+C_{1} \sum_{k=2}^{\infty}\left(\frac{\mathcal{T}_{i}}{\mathcal{T}_{n}}\right)^{k} \frac{x^{-k \gamma}}{k !} \int_{d}^{\frac{\mathcal{T}_{n}}{\mathcal{T}_{i}} x^{\gamma}} y^{k-\alpha-1+\delta} \mathrm{d} y \\
& \leq 1+\frac{\mu}{x^{\gamma}} \frac{\mathcal{T}_{i}}{\mathcal{T}_{n}}+\frac{1}{x^{2 \gamma}} \sum_{k=2}^{\infty}\left(\frac{\mathcal{T}_{i}}{\mathcal{T}_{n}}\right)^{k} \frac{d^{k}}{k !}+C_{2}\left(\frac{\mathcal{T}_{i}}{x^{\gamma} \mathcal{T}_{n}}\right)^{\alpha-\delta} \sum_{k=2}^{\infty} \frac{1}{k !} \\
& \leq 1+\frac{1}{x^{\gamma}} \frac{C_{3}}{\mathcal{T}_{n}}\left(\mu+\frac{C_{3}}{x^{\gamma((\alpha-\delta-1) \wedge 1)}}\right) .
\end{aligned}
$$

Since $C_{3}$ does not depend on $x$ we get that there exits an $x_{0}$ such that for $x>x_{0}$

$$
\begin{aligned}
\sum_{i=1}^{n-1} \log \left(\int_{0}^{\frac{\mathcal{T}_{n}}{\mathcal{T}_{i}} x^{\gamma}} e^{\frac{\mathcal{T}_{i}}{\mathcal{T}_{n} x \gamma} x_{i}} f\left(x_{i}\right) \mathrm{d} x_{i}\right) & \leq \sum_{i=1}^{n-1} \log \left(1+\frac{1}{x^{\gamma}} \frac{\mathcal{T}_{i}}{\mathcal{T}_{n}} \mu\left(1+\frac{c_{2}}{2}\right)\right) \\
& \leq \frac{\mu\left(1+\frac{c_{2}}{2}\right)}{x^{\gamma}} \sum_{i=1}^{n-1} \frac{\mathcal{T}_{i}}{\mathcal{T}_{n}} \leq \frac{1+\frac{c_{2}}{2}}{1+c_{2}} \frac{a x}{x^{\gamma}} .
\end{aligned}
$$

Since $x>c_{1} n$ and $\alpha>1$ we get that

$$
\begin{aligned}
\mathbb{P}\left(\sum_{i=1}^{n-1} \frac{\mathcal{T}_{i}}{\mathcal{T}_{n}} X_{i}>a x, \frac{\mathcal{T}_{i}}{\mathcal{T}_{n}} X_{i} \leq x^{\gamma}\right) & \leq \exp \left(-a x^{1-\gamma}\left(1-\frac{1+\frac{c_{2}}{2}}{1+c_{2}}\right)\right) \\
& \leq \exp \left(-\frac{a c_{2}}{2\left(1+c_{2}\right)} x^{1-\gamma}\right)=o(x f(x))=o(n f(x)) .
\end{aligned}
$$

Lemma A.3. Let $X_{1}, X_{2}, \ldots$ be regularly varying with regularly varying density $f$ and index $\alpha>1$. Denote with $\mu=\mathbb{E}\left[X_{1}\right]$. Let, $c_{1}, \kappa>0$ then there exists a constant $C_{1}$ such that

$$
\limsup _{n \rightarrow \infty} \sup _{\sum_{i=1}^{n-1} \frac{\mathcal{T}_{i}}{\mathcal{T}_{n}}>c_{1} n, \mathcal{T}_{i} \leq \mathcal{T}_{n}} \sup _{x>(1+\kappa) \mu \sum_{i=1}^{n-1} \frac{\mathcal{T}_{i}}{\mathcal{T}_{n}}} \frac{\mathbb{P}\left(\sum_{i=1}^{n-1} \frac{\mathcal{T}_{i}}{\mathcal{T}_{n}} X_{i} \in[x, x+1]\right)}{n \mathbb{P}\left(X_{1} \in[x, x+1]\right)} \leq C_{1} .
$$

If further there exists a $\kappa_{1}>0$ and a series $m_{n}>0 \lim _{n \rightarrow \infty} m_{n} / n=1-\epsilon>0$ then there exists a constant $C_{2}$

$$
\begin{array}{ll}
\limsup _{n \rightarrow \infty} & \sup _{\substack{\mathcal{T}_{m_{n}} \\
\mathcal{T}_{n}} 1 / 2, \sum_{i=1}^{n-1} \frac{\mathcal{T}_{i}}{\mathcal{T}_{n}}>c_{1} n, \mathcal{T}_{i} \leq \mathcal{T}_{n}}(1+\kappa) \mu \sum_{i=1}^{n-1} \frac{\mathcal{T}_{i}}{\mathcal{T}_{n}}<x<M \mu \sum_{i=1}^{n-1} \frac{\mathcal{T}_{i}}{\mathcal{T}_{n}} \\
\frac{\mathbb{P}\left(\sum_{m_{n}+1}^{n} \frac{\mathcal{T}_{i}}{\mathcal{T}_{n}} X_{i}>\left(\mu+\kappa_{1}\right) \sum_{m_{1}+1}^{n} \frac{\mathcal{T}_{i}}{\mathcal{T}_{n}}, \sum_{i=1}^{n-1} \frac{\mathcal{T}_{i}}{\mathcal{T}_{n}} X_{i} \in[x, x+1]\right)}{\epsilon n \mathbb{P}\left(X_{1} \in[x, x+1]\right)} \leq C_{2} .
\end{array}
$$


Proof. At first note that $\mathbb{P}\left(X_{1} \in[x, x+1]\right) \sim f(x)$. Choose a $0<\gamma<1$ such that $2+\alpha<(2 \alpha+1) \gamma$ and $1<\gamma \alpha$. Denote with $B_{i}=\left\{\frac{\mathcal{T}_{i}}{\mathcal{T}_{n}} X_{i} \leq x^{\gamma}\right\}$. We have

$$
\begin{aligned}
& \mathbb{P}\left(\sum_{i=1}^{n-1} \frac{\mathcal{T}_{i}}{\mathcal{T}_{n}} X_{i} \in[x, x+1]\right) \leq \mathbb{P}\left(\sum_{i=1}^{n-1} \frac{\mathcal{T}_{i}}{\mathcal{T}_{n}} X_{i} \in[x, x+1], B_{i} \forall i\right) \\
& +\sum_{k=1}^{n-1} \mathbb{P}\left(\sum_{i=1}^{n-1} \frac{\mathcal{T}_{i}}{\mathcal{T}_{n}} X_{i} \in[x, x+1], B_{k}^{c}, B_{i} i \neq k\right) \\
& +\sum_{l, k=1}^{n-1} \mathbb{P}\left(\sum_{i=1}^{n-1} \frac{\mathcal{T}_{i}}{\mathcal{T}_{n}} X_{i} \in[x, x+1], B_{k}^{c}, B_{l}^{c}, B_{i} i \neq k, l\right)
\end{aligned}
$$

From Lemma A.2 we get

$$
\mathbb{P}\left(\sum_{i=1}^{n-1} \frac{\mathcal{T}_{i}}{\mathcal{T}_{n}} X_{i} \in[x, x+1], B_{i} \forall i\right)=o(n f(x)) .
$$

Further we get that

$$
\begin{aligned}
& \mathbb{P}\left(\sum_{i=1}^{n-1} \frac{\mathcal{T}_{i}}{\mathcal{T}_{n}} X_{i} \in[x, x+1], B_{k}^{c}, B_{l}^{c}\right) \\
& \leq \mathbb{E}\left[\int_{\frac{\mathcal{T}_{n}}{\mathcal{T}_{k}}\left(x-\sum_{i \neq k} \frac{\mathcal{T}_{i}}{\mathcal{T}_{n}} X_{i}\right)} f(y) \mathrm{d} y 1_{\left\{x+1-\sum_{i \neq k} \frac{\mathcal{T}_{i}}{\mathcal{T}_{n}} X_{i}>x^{\gamma}, \frac{\mathcal{T}_{l}}{\mathcal{T}_{n}} X_{l}>x^{\gamma}\right\}}\right] \\
& \lesssim \mathbb{E}\left[\frac{\mathcal{T}_{n}}{\mathcal{T}_{k}} f\left(\frac{\mathcal{T}_{n}}{\mathcal{T}_{k}}\left(x-\sum_{i \neq k} \frac{\mathcal{T}_{i}}{\mathcal{T}_{n}} X_{i}\right)\right) 1_{\left\{x+1-\sum_{i \neq k} \frac{\mathcal{T}_{i}}{\mathcal{T}_{n}} X_{i}>x^{\gamma}, \frac{\mathcal{T}_{l}}{\mathcal{T}_{n}} X_{l}>x^{\gamma}\right\}}\right] \\
& \lesssim \mathbb{E}\left[\frac{\mathcal{T}_{n}}{\mathcal{T}_{k}} f\left(\frac{\mathcal{T}_{n}}{\mathcal{T}_{k}}\left(x^{\gamma}-1\right)\right) 1_{\left\{x+1-\sum_{i \neq k} \frac{\mathcal{T}_{i}}{\mathcal{T}_{n}} X_{i}>\frac{\mathcal{T}_{k}}{\mathcal{T}_{n}} x^{\gamma}, X_{l}>x^{\gamma}\right\}}\right] \\
& \lesssim \frac{\mathcal{T}_{n}}{\mathcal{T}_{k}} f\left(\frac{\mathcal{T}_{n}}{\mathcal{T}_{k}}\left(x^{\gamma}-1\right)\right) \bar{F}\left(\frac{\mathcal{T}_{n}}{\mathcal{T}_{k}} x^{\gamma}\right) \\
& \leq c f\left(x^{\gamma}\right) \bar{F}\left(x^{\gamma}\right) \text {, }
\end{aligned}
$$

for some $c>1$ and we used Potter bounds in the last equality. Since $x>c_{1} \mu n$,

$$
\frac{n^{2} f\left(x^{\gamma}\right) \bar{F}\left(x^{\gamma}\right)}{n f(x)} \leq \frac{1}{c_{1} \mu} \frac{x f\left(x^{\gamma}\right) \bar{F}\left(x^{\gamma}\right)}{f(x)}
$$

and the last term is regularly varying with index $2-(2 \gamma-1) \alpha-\gamma<0$, we are left with (9). Denote with $Z=\sum_{i \neq k} \frac{\mathcal{T}_{i}}{\mathcal{T}_{n}} X_{i}$. Then

$$
\begin{aligned}
& \mathbb{P}\left(\sum_{i=1}^{n-1} \frac{\mathcal{T}_{i}}{\mathcal{T}_{n}} X_{i} \in[x, x+1], B_{k}^{c}, B_{i} i \neq k\right) \\
& =\mathbb{P}\left(\sum_{i=1}^{n-1} \frac{\mathcal{T}_{i}}{\mathcal{T}_{n}} X_{i} \in[x, x+1], Z>\frac{1+\kappa / 2}{1+\kappa} x, B_{k}^{c}, B_{i} i \neq k\right)
\end{aligned}
$$




$$
+\mathbb{P}\left(\sum_{i=1}^{n-1} \frac{\mathcal{T}_{i}}{\mathcal{T}_{n}} X_{i} \in[x, x+1], Z \leq \frac{1+\kappa / 2}{1+\kappa} x, B_{k}^{c}, B_{i} i \neq k\right) .
$$

Since

$$
\frac{1+\kappa / 2}{1+\kappa} x>(1+\kappa / 2) \mu \sum_{i=1}^{n} \frac{\mathcal{T}_{i}}{\mathcal{T}_{n}}
$$

we get by Lemma A.2 that

$$
\begin{aligned}
& \mathbb{P}\left(\sum_{i=1}^{n-1} \frac{\mathcal{T}_{i}}{\mathcal{T}_{n}} X_{i} \in[x, x+1], Z>\frac{1+\kappa / 2}{1+\kappa} x, B_{k}^{c}, B_{i} i \neq k\right) \\
& \leq \mathbb{P}\left(X_{i}>\frac{\mathcal{T}_{n}}{\mathcal{T}_{i}} x^{\gamma}\right) \mathbb{P}\left(Z>\frac{1+\kappa / 2}{1+\kappa} x, B_{i} i \neq k\right) \\
& =o\left(n f(x) \bar{F}\left(x^{\gamma}\right)\right)=o(f(x)) .
\end{aligned}
$$

Since $\bar{F}\left(x^{\gamma}\right)=o(n)$. Finally we have that

$$
\begin{aligned}
& \mathbb{P}\left(\sum_{i=1}^{n-1} \frac{\mathcal{T}_{i}}{\mathcal{T}_{n}} X_{i} \in[x, x+1], Z \leq \frac{1+\kappa / 2}{1+\kappa} x, B_{k}^{c}, B_{i} i \neq k\right) \\
& =\int_{0}^{\frac{1+\kappa / 2}{1+\kappa} x} F\left(\frac{\mathcal{T}_{n}}{\mathcal{T}_{k}}(x-z)\right)-\bar{F}\left(\frac{\mathcal{T}_{n}}{\mathcal{T}_{k}}(x-z+1)\right) \mathrm{d} F_{Z}(z) \\
& \lesssim \int_{0}^{\frac{1+\kappa / 2}{1+\kappa} x} \frac{\mathcal{T}_{n}}{\mathcal{T}_{k}} f\left(\frac{\mathcal{T}_{n}}{\mathcal{T}_{k}}(x-z)\right) \mathrm{d} F_{Z}(z) \\
& \lesssim f(x) \int_{0}^{\frac{1+\kappa / 2}{1+\kappa} x} \frac{f(x-z)}{f(x)} \mathrm{d} F_{Z}(z) \\
& \lesssim\left(\frac{\kappa}{2(1+\kappa)}\right)^{-\alpha} f(x),
\end{aligned}
$$

where the last inequality follows since regularly varying functions are asymptotically monotone. This proves (7). Since from Lemma A.2 we get that

$$
\begin{aligned}
& \mathbb{P}\left(\sum_{m_{1}+1}^{n} \frac{\mathcal{T}_{i}}{\mathcal{T}_{n}} X_{i}>\left(\mu+\kappa_{1}\right) \sum_{m_{1}+1}^{n} \frac{\mathcal{T}_{i}}{\mathcal{T}_{n}}, B_{i} i>m_{n}\right) \\
& =o\left(\left(n-m_{n}\right) f\left(\left(\mu+\kappa_{1}\right) \sum_{m_{1}+1}^{n} \frac{\mathcal{T}_{i}}{\mathcal{T}_{n}}\right)\right)=o(\epsilon n f(\epsilon x /(2 M)))=o(n f(x)) .
\end{aligned}
$$

also (8) can be proved along the same lines (one only has to assume $B_{i}$ for $i>m_{n}$ ).

\section{B Auxiliary results}

In this section we show some basic Lemmas that are needed in several parts of the proofs. Nevertheless one should note that none of these results are surprising and the proofs use basically only standard techniques, so one may find the same or similar results and methods 
in other papers but since we don't have a good reference and for reasons of completeness we provide the proofs.

The following Lemma is basically a restatement of the results in [1, Section 4] which basically provides a lower bound for ruin probabilities in quite general risk models. Since [1] uses a different model we provide also a proof here.

Lemma B.1. Assume that $S_{t}=\sum_{i=1}^{N_{t}} X_{\mathcal{T}_{i}}-p(t)$ ( $N_{t}$ is Poisson process with intensity $\lambda_{t}$ assume that $X_{t}$ and $N_{t}$ are independent and that there exists a continuous function $c(t)>0$ such that $S_{t-} \lesssim-c(t)$ a.s.. Then for every $\epsilon>0$ there exists an $K>0$ - such that for $u>K$

$$
\mathbb{P}\left(\sup _{0 \leq t} S_{t}>u\right) \geq(1-\epsilon) \int_{0}^{\infty} \lambda_{t} \mathbb{P}\left(X_{t}>u+K+(1+\epsilon) c(t)\right) \mathrm{d} t
$$

as $u \rightarrow \infty$

Proof. Since $S_{t-} \sim-c_{t}$ a.s. for every $\epsilon>0$ there exists and $K>0$ such that for the set

$$
A_{\epsilon}=\left\{S_{t-}<K-(1+\epsilon) c_{t}, \forall t>0\right\}
$$

$\mathbb{P}\left(A_{\epsilon}\right)>1-\epsilon$. Denote with $\tau(u)=\inf \left\{t: S_{t}>u\right\}$ and with $\bar{F}_{t}(x)=\mathbb{P}\left(X_{t}>x\right)$. On the set $A_{\epsilon}$ we get that for $u>K, \tau(u)=\infty$ and hence

$$
\begin{aligned}
\mathbb{P}\left(\sup _{0 \leq t} S_{t}>u\right) & \geq \int_{0}^{\infty} \lambda_{t} \mathbb{E}\left[\bar{F}_{t}\left(u-S_{t-}\right) 1_{\{\tau(u)>t\}}\right] \mathrm{d} t \\
& \geq \int_{0}^{\infty} \lambda_{t} \mathbb{E}\left[\bar{F}_{t}\left(u-S_{t-}\right) 1_{\left\{A_{\epsilon}\right\}} 1_{\{\tau(u)>t\}}\right] \mathrm{d} t \\
& \geq \int_{0}^{\infty} \lambda_{t} \mathbb{E}\left[\bar{F}_{t}\left(u+K+(1+\epsilon) c_{t}\right) 1_{\left\{A_{\epsilon}\right\}}\right] \mathrm{d} t \\
& \geq(1-\epsilon) \int_{0}^{\infty} \lambda_{t} \bar{F}_{t}\left(u+K+(1+\epsilon) c_{t}\right) \mathrm{d} t .
\end{aligned}
$$

The following Lemma is just an application of Chebyshev inequality. We manly give it here because we need it in different places in the proofs.

Lemma B.2. Let $X$ be Erlang $(\lambda, k)$ then for $\epsilon>0$ there exists a $\kappa_{\epsilon}>0$ such that for all $k$

$$
\begin{aligned}
& \mathbb{P}(X \leq(1-\epsilon) k / \lambda) \leq e^{-\kappa_{\epsilon} k} \\
& \mathbb{P}(X>(1+\epsilon) k / \lambda) \leq e^{-\kappa_{\epsilon} k} .
\end{aligned}
$$

Proof. Choose an $s>0$ such that

$$
1-\epsilon<\frac{\log (1+s)}{s}
$$

and set $\kappa_{\epsilon}^{l}=\log (1+s)-s(1-\epsilon)$ then

$$
\mathbb{P}(X \leq(1-\epsilon) k / \lambda) \leq e^{s(1-\epsilon) k} \mathbb{E}\left[e^{-s \lambda X}\right]=e^{(s(1-\epsilon)-\log (1+s)) k}=e^{-\kappa_{\epsilon}^{l} k} .
$$


Similarly we get for

$$
1+\epsilon>\frac{-\log (1+s)}{s}
$$

and $\kappa_{\epsilon}^{u}=s(1+\epsilon)+\log (1-s)$ that

$$
\mathbb{P}(X>(1+\epsilon) k / \lambda) \leq e^{-s(1+\epsilon) k} \mathbb{E}\left[e^{s \lambda X}\right]=e^{-(s(1+\epsilon)+\log (1-s)) k}=e^{-\kappa_{\epsilon}^{u} k} .
$$

The following Lemma is equivalent to the well known asymptotic of $\int_{c u}^{u} \bar{F}(u-x) g(x) \mathrm{d} x$ when $g(x) \leq c f(x)$ and $f(x)$ is regularly varying. We only state it because we need it in two places in the proof.

Lemma B.3. Assume that $X_{1}, \ldots, X_{n}$ are independent positive random variables and $X_{n}$ has distribution function $F$ that is regularly varying with index $\alpha$ and has a regularly varying density $f$. Further assume that there exists a set $B$, independent of $X_{n}$ and that for some $C_{u, n}>0$ and $0<c<1$ and all $c u<x<u$

$$
\mathbb{P}\left(\sum_{i=1}^{n-1} X_{i} \in[x, x+1), B\right) \leq C_{u, n} \mathbb{P}\left(X_{n} \in[x, x+1)\right) .
$$

Then we have

$$
\begin{aligned}
& \mathbb{P}\left(\sum_{i=1}^{n} X_{i}>u, \sum_{i=1}^{n-1} X_{i} \in[c u, u), B\right) \\
& \quad \lesssim\left(1+c^{-\alpha}\right) C_{u, n} \sup _{x \geq 0} \frac{\bar{F}(x)}{\bar{F}(x+1)} \int_{0}^{(1-c) u} \bar{F}(x) \mathrm{d} x f(u),
\end{aligned}
$$

where the asymptotic is uniformly in $n$ and the distribution of $X_{1}, \ldots, X_{n-1}$.

Proof. Denote with $m=\lceil(1-c) u\rceil$

$$
\begin{aligned}
& \mathbb{P}\left(\sum_{i=1}^{n} X_{i}>u, \sum_{i=1}^{n-1} X_{i} \in[c u, u), B\right) \\
& \leq \sum_{k=1}^{m} \mathbb{P}\left(\sum_{i=1}^{n} X_{i}>u, \sum_{i=1}^{n-1} X_{i} \in[c u+k-1, c u+k), B\right) \\
& \leq \sum_{k=1}^{m} \mathbb{P}\left(X_{n}>u-c u-k, \sum_{i=1}^{n-1} X_{i} \in[c u+k-1, c u+k), B\right) \\
& =\sum_{k=1}^{m} \mathbb{P}\left(X_{n}>u-c u-k\right) \mathbb{P}\left(\sum_{i=1}^{n-1} X_{i} \in[c u+k-1, c u+k), B\right) \\
& \leq C_{u, n} \sup _{x>0} \frac{\bar{F}(x)}{\bar{F}(x+1)} \sum_{k=1}^{m} \mathbb{P}\left(X_{n}>u-c u-k+1\right) \mathbb{P}\left(X_{n} \in[c u+k-1, c u+k)\right) \\
& \leq C_{u, n} \sup _{x>0} \frac{\bar{F}(x)}{\bar{F}(x+1)} \sum_{k=1}^{m} \int_{c u+k-1}^{c u+k} \bar{F}(u-x) \mathrm{d} F(x)
\end{aligned}
$$




$$
\leq C_{u, n} \sup _{x>0} \frac{\bar{F}(x)}{\bar{F}(x+1)}\left(\int_{c u}^{u+1} \bar{F}(u-x) \mathrm{d} F(x)\right) .
$$

Note that

$$
\int_{u}^{u+1} \bar{F}(u-x) \mathrm{d} F(x)=\int_{u}^{u+1} \mathrm{~d} F(x) \sim f(u) .
$$

And the lemma follows from

$$
\int_{c u}^{u} \bar{F}(u-x) \mathrm{d} F(x)=\int_{0}^{(1-c) u} f(u-x) \bar{F}(x) \mathrm{d} x \lesssim f(u) c^{-\alpha} \int_{0}^{(1-c) u} \bar{F}(x) \mathrm{d} x .
$$

The following Lemma is basically a standard version of the weak law of large numbers of weighted random variables, we only give a proof to point out that the result is somehow uniform in the weights.

Lemma B.4. Assume that $X_{1}, X_{2}, \ldots$ are i.i.d. random variables with common distribution function $F$ that is regularly varying with index $\alpha>1$ and mean $\mu$. Further let $\kappa, C>0$ then

$$
\lim _{n \rightarrow \infty} \sup _{0 \leq c_{i} \leq 1, \sum_{i=1}^{n} c_{i}>C n} \mathbb{P}\left(\sum_{i=1}^{n} c_{i} X_{i}>(1+\kappa) \mu \sum_{i=1}^{n} c_{i}\right)=0 .
$$

Proof. At first note that

$$
\lim _{n \rightarrow \infty} \mathbb{P}\left(\sum_{i=1}^{n} c_{i} X_{i}>(1+\kappa) \mu \sum_{i=1}^{n} c_{i}, \exists X_{i}>n\right) \leq \lim _{n \rightarrow \infty} \sum_{i=1}^{n} \mathbb{P}\left(X_{i}>n\right)=\lim _{n \rightarrow \infty} n \bar{F}(n) \rightarrow 0 .
$$

Denote with $\mu_{n}=\mathbb{E}\left[X_{1} 1_{\left\{X_{1} \leq n\right\}}\right]$. We get with Chebyshev

$$
\begin{aligned}
& \mathbb{P}\left(\sum_{i=1}^{n} c_{i} X_{i}>(1+\kappa) \mu \sum_{i=1}^{n} c_{i}, \forall X_{i} \leq n\right) \\
& \leq \mathbb{P}\left(\sum_{i=1}^{n} c_{i} X_{i}>(1+\kappa) \mu_{n} \sum_{i=1}^{n} c_{i}, \forall X_{i} \leq n\right) \\
& =\mathbb{P}\left(\sum_{i=1}^{n} c_{i}\left(X_{i}-\mu_{n}\right)>\kappa \mu_{n} \sum_{i=1}^{n} c_{i}, \forall X_{i} \leq n\right) \\
& \leq \frac{1}{\left(\kappa \mu_{n} \sum_{i=1}^{n} c_{i}\right)^{2}} \sum_{i=1}^{n} \mathbb{E}\left[\left(c_{i}\left(X_{i}-\mu_{n}\right)\right)^{2} 1_{\left\{X_{i} \leq n\right\}}\right] \\
& \leq \frac{\mu_{n}^{2} \sum_{i=1}^{n} c_{i}^{2}+\mathbb{E}\left[X_{i}^{2} 1_{\left\{X_{i} \leq n\right\}}\right] \sum_{i=1}^{n} c_{i}^{2}}{\left(\kappa \mu_{n} \sum_{i=1}^{n} c_{i}\right)^{2}} \\
& \lesssim \frac{\mu_{n}^{2}+\mathbb{E}\left[X_{i}^{2} 1_{\left\{X_{i} \leq n\right\}}\right]}{\left(\kappa \mu_{n}\right)^{2} \sum_{i=1}^{n} c_{i}} \lesssim \frac{\mu_{n}^{2}+\mathbb{E}\left[X_{i}^{2} 1_{\left\{X_{i} \leq n\right\}}\right]}{\left(\kappa \mu_{n}\right)^{2} C n} .
\end{aligned}
$$

Since $X_{1}$ is regularly varying with index $\alpha>1$ we get by Karamata's Theorem that $\mathbb{E}\left[X_{i}^{2} 1_{\left\{X_{i} \leq n\right\}}\right]=o(n)$ which proves the Lemma. 
Lemma B.5. Assume that $\frac{\mathcal{T}_{n}}{\lambda n} \rightarrow 1$ a.s. and the $X_{i}$ are i.i.d. with mean $\mu$ then

$$
\sum_{i=1}^{n} \mathcal{T}_{i} X_{i} \sim \lambda \mu \sum_{i=1}^{n} i \quad \text { a.s. }
$$

Proof. We have to show that for every $\epsilon>0$ and $\delta>0$ there exists an $n_{0}$ such that

$$
\mathbb{P}\left(\sup _{n \geq n_{0}}\left|\frac{\sum_{i=1}^{n} \mathcal{T}_{i} X_{i}}{\lambda \mu \sum_{i=1}^{n} i}-1\right|>\epsilon\right) \leq \delta .
$$

Therefore choose an $\epsilon_{1}$ such that

$$
1-\epsilon<\left(1-\epsilon_{1}\right)^{3}<\epsilon_{1}+\left(1+\epsilon_{1}\right)^{2}<1+\epsilon .
$$

and $n_{1}>0, n_{2}>0$ and $n_{3}>n_{1}+n_{2}$ such that

$$
\frac{\sum_{i=n_{1}}^{n_{3}} i}{\sum_{i=1}^{n_{3}} i} \geq 1-\epsilon_{1}
$$

and the sets

$$
\begin{aligned}
& B_{1}=B_{1}\left(n_{1}, \epsilon_{1}\right)=\left\{\sup _{n \geq n_{1}}\left|\frac{\mathcal{T}_{n}}{\lambda n}-1\right|>\epsilon_{1}\right\}, \\
& B_{2}=B_{2}\left(n_{1}, n_{2}, \epsilon_{1}\right)=\left\{\sup _{n \geq n_{2}}\left|\frac{\sum_{i=n_{1}}^{n_{1}+n} i X_{i}}{\mu \sum_{i=n_{1}}^{n_{1}+n} i}-1\right|>\epsilon_{1}\right\}, \\
& B_{3}=B_{3}\left(n_{1}, n_{3}, \epsilon_{1}\right)=\left\{\frac{\sum_{i=1}^{n_{1}} \mathcal{T}_{i} X_{i}}{\lambda \mu \sum_{i=1}^{n_{3}} i}>\epsilon_{1}\right\},
\end{aligned}
$$

fulfill $\mathbb{P}\left(B_{i}\right) \leq \delta / 3$. This can be done since $\frac{\mathcal{T}_{n}}{\lambda n} \rightarrow 1$ a.s and [13] proves that

$$
\frac{\sum_{i=n_{1}}^{n_{1}+n} i X_{i}}{\mu \sum_{i=n_{1}}^{n_{1}+n} i} \rightarrow 1 \quad \text { a.s.. }
$$

On $B_{1}^{c} \cap B_{2}^{c} \cap B_{3}^{c}$ we have that for $n \geq n_{3}$

$$
\begin{aligned}
\frac{\sum_{i=1}^{n} \mathcal{T}_{i} X_{i}}{\lambda \mu \sum_{i=1}^{n} i}-1 & \leq \frac{\sum_{i=1}^{n_{1}} \mathcal{T}_{i} X_{i}}{\lambda \mu \sum_{i=1}^{n} i}+\frac{\sum_{i=n_{1}}^{n} \mathcal{T}_{i} X_{i}}{\lambda \mu \sum_{i=1}^{n} i}-1 \\
& \leq \epsilon_{1}+\left(1+\epsilon_{1}\right) \frac{\sum_{i=n_{1}}^{n} i}{\sum_{i=1}^{n} i} \frac{\sum_{i=n_{1}}^{n} i X_{i}}{\mu \sum_{i=n_{1}}^{n} i}-1 \\
& \leq \epsilon_{1}+\left(1+\epsilon_{1}\right)^{2}-1 \leq \epsilon
\end{aligned}
$$

with the same arguments we get that

It follows that

$$
\frac{\sum_{i=1}^{n} \mathcal{T}_{i} X_{i}}{\lambda \mu \sum_{i=1}^{n} i}-1 \geq\left(1-\epsilon_{1}\right)^{3}-1 \geq-\epsilon .
$$

and hence

$$
\left\{\sup _{n \geq n_{3}}\left|\frac{\sum_{i=1}^{n} \mathcal{T}_{i} X_{i}}{\lambda \mu \sum_{i=1}^{n} i}-1\right|>\epsilon\right\} \subseteq B_{1} \cup B_{2} \cup B_{3}
$$

$$
\mathbb{P}\left(\sup _{n \geq n_{3}}\left|\frac{\sum_{i=1}^{n} \mathcal{T}_{i} X_{i}}{\lambda \mu \sum_{i=1}^{n} i}-1\right|>\epsilon\right) \leq \delta .
$$




\section{References}

[1] S. Asmussen. On the ruin problem for some adapted premium rules. In In Kalashnikov, V. V. ES Andronov, A. M. (eds.), Probabilistic Analysis of Rare Events: Theory and Problems of Safety, Insurance and Ruin., pages 1-15. Riga Aviations University, 1999.

[2] S. Asmussen and R. Biard. Ruin probabilities for a regenerative poisson gap generated risk process. European Actuarial Journal, 1:3-22, 2011. 10.1007/s13385-011-0002-8.

[3] S. Asmussen, L. Fløe Henriksen, and C. Klüppelberg. Large claims approximations for risk processes in a Markovian environment. Stochastic Process. Appl., 54(1):29-43, 1994.

[4] S. Asmussen and T. Rolski. Risk theory in a periodic environment: the CramérLundberg approximation and Lundberg's inequality. Math. Oper. Res., 19(2):410433, 1994.

[5] S. Asmussen, H. Schmidli, and V. Schmidt. Tail probabilities for non-standard risk and queueing processes with subexponential jumps. Adv. in Appl. Probab., 31(2):422447, 1999.

[6] M. Bargès, H. Cossette, S. Loisel, and É. Marceau. On the moments of aggregate discounted claims with dependence introduced by a FGM copula. Astin Bull., 41(1):215$277,2011$.

[7] N. H. Bingham, C. M. Goldie, and J. L. Teugels. Regular variation, volume 27 of Encyclopedia of Mathematics and its Applications. Cambridge University Press, Cambridge, 1989.

[8] A. Castañer, M. Mercé Claramunt, M. Gathy, C. Lefévre, and M. Mármol. Ruin problems for a discrete time risk model with non-homogeneous conditions. Scandinavian Actuarial Journal, 0(0):1-20, 0 .

[9] D. Denisov, A. B. Dieker, and V. Shneer. Large deviations for random walks under subexponentiality: the big-jump domain. Ann. Probab., 36(5):1946-1991, 2008.

[10] P. Embrechts, C. Klüppelberg, and T. Mikosch. Modelling extremal events, for insurance and finance, volume 33 of Applications of Mathematics (New York). SpringerVerlag, Berlin, 1997.

[11] H. U. Gerber. On additive premium calculation principles. Astin Bull., 7(3):215-222, 1974.

[12] IPCC. Emissions scenarios, http://www.ipcc.ch/pdf/special-reports/emissions_scenarios.pdf, 2000.

[13] B. Jamison, S. Orey, and W. Pruitt. Convergence of weighted averages of independent random variables. Z. Wahrscheinlichkeitstheorie und Verw. Gebiete, 4:40-44, 1965. 
[14] V. Kalashnikov and D. Konstantinides. Ruin under interest force and subexponential claims: a simple treatment. Insurance Math. Econom., 27(1):145-149, 2000.

[15] J. Li, Q. Tang, and R. Wu. Subexponential tails of discounted aggregate claims in a time-dependent renewal risk model. Adv. in Appl. Probab., 42(4):1126-1146, 2010.

[16] J. Neslehova, P. Embrechts, and V. Chavez-Demoulin. Infinite mean models and the lda for operational risk. Journal of Operational Risk, 1(1):3-25, 2006.

[17] J. Paulsen. Risk theory in a stochastic economic environment. Stochastic Process. Appl., 46(2):327-361, 1993. 